\title{
Best proximity and fixed point results for cyclic multivalued mappings under a generalized contractive condition
}

\author{
Manuel De la Sen ${ }^{*}$, Shyam Lal Singh ${ }^{2}$, Madjid Eshaghi Gordji ${ }^{3}$, Asier Ibeas ${ }^{4}$ and Ravi P Agarwal ${ }^{5,6}$
}

\section{"Correspondence:}

manuel.delasen@ehu.es

${ }^{1}$ Institute of Research and

Development of Processes,

University of Basque Country, Campus of Leioa (Bizkaia) - Aptdo.

644 - Bilbao, Bilbao, 48080, Spain Full list of author information is

available at the end of the article

\begin{abstract}
This paper is devoted to investigating the existence of fixed points and best proximity points of multivalued cyclic self-mappings in metric spaces under a generalized contractive condition involving Hausdorff distances. Some background results for cyclic self-mappings or for multivalued self-mappings in metric fixed point theory are extended to cyclic multivalued self-mappings. An example concerned with the global stability of a time-varying discrete-time system is also discussed by applying some of the results obtained in this paper. Such an example includes the analysis with numerical simulations of two particular cases which are focused on switched discrete-time control and integrate the associate theory in the context of multivalued mappings.
\end{abstract}

MSC: $47 \mathrm{H} 10 ; 55 \mathrm{M} 20 ; 54 \mathrm{H} 25$

Keywords: best proximity points; cyclic self-mappings; fixed points; metric space; multi-control; multivalued self-mappings; uniform convex Banach space

\section{Introduction}

Important attention is being devoted to investigation of fixed point theory for singlevalued and multivalued mappings concerning some relevant properties like, for instance, stability of the iterations, fixed points of contractive and nonexpansive self-mappings and the existence of either common or coupled fixed points of several multivalued mappings or operators. See, for instance, [1-24] and references therein. Related problems concerning the computational aspects of iterative calculations and best approximations based on fixed point theory have been also investigated. See, for instance, $[21-23,25,26]$ and some references therein. On the other hand, a fixed point result for partial metric spaces and partially ordered metric spaces can be found in [27-30] and [4, 15, 31, 32], respectively, and references therein.

This paper is devoted to the investigation of some properties of fixed point and best proximity point results for multivalued cyclic self-mappings under a general contractivetype condition based on the Hausdorff metric between subsets of a metric space [1-3, $33,34]$. This includes, as a particular case, contractive single-valued self-mappings [1-3, 25,33-36], and similar problems for cyclic (strictly contractive or not) self-mappings [3537] as well. Some previous results on multivalued contractions are retaken by generalizing the contractive condition and extended to cyclic multivalued self-mappings by extending the results of Đorić and Lazović in [1] (then being extended in [6] concerning results on 
common fixed points of a pair of multivalued maps in a complete metric space) which are based on previous Suzuki et al. and Ćirić's results for single-valued self-mappings in some background literature papers. See, for instance, $[2,3,33,34]$ and references therein. Through this paper, we consider a metric space $(X, d)$ and a multivalued 2-cyclic selfmapping $T: A \cup B \rightarrow A \cup B$ (being simply referred to as a multivalued cyclic self-mapping in the sequel), where $A$ and $B$ are nonempty closed subsets of $X$, so that $T(A) \subseteq B$ and $T(B) \subseteq A$ and $D=\operatorname{dist}(A, B) \geq 0$. Let us consider the subset of the set of real numbers $\mathbf{R}_{0+}=\mathbf{R}_{+} \cup\{0\}=\{z \geq 0: z \in \mathbf{R}\}, \mathbf{R}_{+}=\{z>0: z \in \mathbf{R}\}$, let the symbols ' $\vee$ ' and ' $\wedge$ ' denote the logic disjunction ('or') and conjunction ('and'), and define the functions $M:(A \cup B) \times(B \cup$ $A) \times[0,1) \times \Delta \rightarrow \mathbf{R}_{0+}$ and $\varphi:(A \cup B) \times(B \cup A) \times[0,1) \times \Delta \rightarrow(0,1]$ as follows:

$$
\begin{aligned}
& M(x, y, K, \alpha, \beta)=\max \left[M_{1}(x, y, K), M_{2}(x, y, \alpha, \beta)\right], \\
& M_{1}(x, y, K)=K \max \{d(x, y), d(x, T x), d(y, T y), 1 / 2(d(x, T y)+d(y, T x))\}, \\
& M_{2}(x, y, \alpha, \beta)=\alpha d(x, T x)+\beta d(y, T y), \\
& \varphi(x, y, K, \alpha, \beta) \\
& =\left\{\begin{aligned}
1 & \text { if }\left(\left[(\alpha, \beta) \in \Delta_{1} \backslash\left(\Delta_{3} \cup \Delta_{4}\right)\right] \vee\left[(\alpha, \beta) \in \Delta_{2} \backslash\left(\Delta_{3} \cup \Delta_{4}\right)\right]\right) \\
1-\beta & \text { if }\left(\left[(\alpha, \beta) \in \Delta_{3}\right]\right) \wedge\left(M_{2}(x, y, \alpha, \beta)>M_{1}(x, y, K)\right), \\
\frac{1-\beta}{1-\beta+\alpha} & \text { if }\left((\alpha, \beta) \in \Delta_{4} \backslash \Delta_{3}\right) \wedge\left(M_{2}(x, y, \alpha, \beta)>M_{1}(x, y, K)\right), \\
1 & \text { if }(0 \leq K<1 / 2) \wedge\left(M_{2}(x, y, \alpha, \beta) \leq M_{1}(x, y, K)\right), \\
1-K & \text { if }(1 / 2 \leq K<1) \wedge\left(M_{2}(x, y, \alpha, \beta) \leq M_{1}(x, y, K)\right) ;
\end{aligned}\right.
\end{aligned}
$$

$\forall x, y \in(A \cup B) \times(B \cup A)$ for some real constants $K \in[0,1),(\alpha, \beta) \in \Delta \subseteq[0,1) \times[0,1)$, where

$$
\begin{aligned}
& \Delta=\{(\alpha, \beta): \alpha \geq 0, \beta \geq 0, \alpha+\beta<1\}, \\
& \Delta_{1}=\{(\alpha, \beta) \in \Delta: \alpha \leq \beta, \alpha(1+\alpha)+\beta<1\}, \\
& \Delta_{2}=\{(\alpha, \beta) \in \Delta: \alpha \geq \beta, \beta(1+\beta)+\alpha<1\}, \\
& \Delta_{3}=\left\{(\alpha, \beta) \in \Delta: \alpha \in(0,1 / 2), \frac{1-\alpha}{2} \geq \beta\right\}, \\
& \Delta_{4}=\{(\alpha, \beta) \in \Delta: \alpha(1+\alpha)+\beta(2-\beta) \geq 1\} .
\end{aligned}
$$

Note that $\varphi:(A \cup B) \times(B \cup A) \times[0,1) \times \Delta \rightarrow(0,1]$ is non-increasing since all its partial derivatives with respect to $K, \alpha, \beta$ exist and are non-positive; $\forall x, y \in(A \cup B) \times(B \cup A)$ and note also that $\Delta$ is the union of the subsets $\Delta_{i} \subset \Delta ; i=1,2,3,4$.

A general contractive condition is then proposed and discussed based on the Hausdorff metric on subsets of a vector space and the constraints (1.1)-(1.4). For this purpose, some preparatory concepts are needed. Let $C L(X)$ be a family of all nonempty and closed subsets of the vector space $X$. If $A, B \in C L(X)$, then we can define $(C L(X), H)$ as the generalized hyperspace of $(X, d)$ equipped with the Hausdorff metric $H: C L(X) \rightarrow \mathbf{R}_{0+}$

$$
H(A, B)=\max \left\{\sup _{x \in A} d(x, B), \sup _{y \in B} d(y, A)\right\}
$$


with $A \subset X$ and $B \subset X$ being nonempty sets. The gap between the nonempty sets $A$ and $B$ is defined by

$$
D=\operatorname{dist}(A, B)=\inf _{x \in A, y \in B} d(x, y)=\inf _{x \in A} d(x, B)=\inf _{y \in B} d(y, A) \geq 0 .
$$

The proposed general contractive condition to be then discussed is

$$
[\varphi(x, y, K, \alpha, \beta) d(x, T x) \leq d(x, y)] \Rightarrow[H(T x, T y) \leq M(x, y, K, \alpha, \beta)+\omega D]
$$

where $T: A \cup B \rightarrow A \cup B$ is a multivalued cyclic self-mapping on the subset $A \cup B$ of $X$, that is, $T(A) \subseteq B$ and $T(B) \subseteq A$, where $(X, d)$ is a complete metric space including the case that $(X,\|\|)$ is a Banach space with a norm-induced metric $d: X \times X \rightarrow \mathbf{R}_{0+}$, so that $(X,\|\|) \equiv(X, d)$ is a complete metric space, is used, subject to (1.1)-(1.4), in the main result Theorem 2.1 below. In this context, $T x$ is the image set through $T$ of any $x \in A \cup B$ which is in $B$, that is, $T x \subset T(A) \subseteq B$ (respectively, $T x \subset T(B) \subseteq A$ ) if $x \in A$ (respectively, if $x \in B$ ). It is inspired by that proposed in [34] for single-valued self-mappings while it generalizes that proposed and discussed in [1] for multivalued self-mappings which is based on the Hausdorff generalized metric.

See Figure 1 with the plots of the various involved sets $\Delta$ and $\Delta_{i} \subset \Delta$ for $i=1,2,3,4$ and some of their relevant subsets in the contractive condition subject to (1.1)-(1.4).

Note that the proposed contractive condition, in fact, considers the worst case, given by the maximum of (1.1), of such a contractive condition of [1], reflected in (1.2a), with one based on a Kannan-type contractive condition associated with the choice of possible distinct values for the constants $\alpha$ and $\beta$, which is reflected in (1.2b) subject to (1.3)-(1.4). In particular, the choice $\alpha=\beta \in[0,1 / 2)$ gives a Kannan-type contractive condition in (1.2b). Note the importance of Kannan-type contractions for single-valued mappings in the sense that a metric space is complete if and only if each Kannan contraction has a unique fixed point $[27,38,39]$. The incorporation of $(1.2 b),(1.3)-(1.4)$ to (1.1) to build the general contractive condition allows an obvious direct generalization of the usual contractive condition, based on the Banach principle combined with a Kannan-type constraint, since both
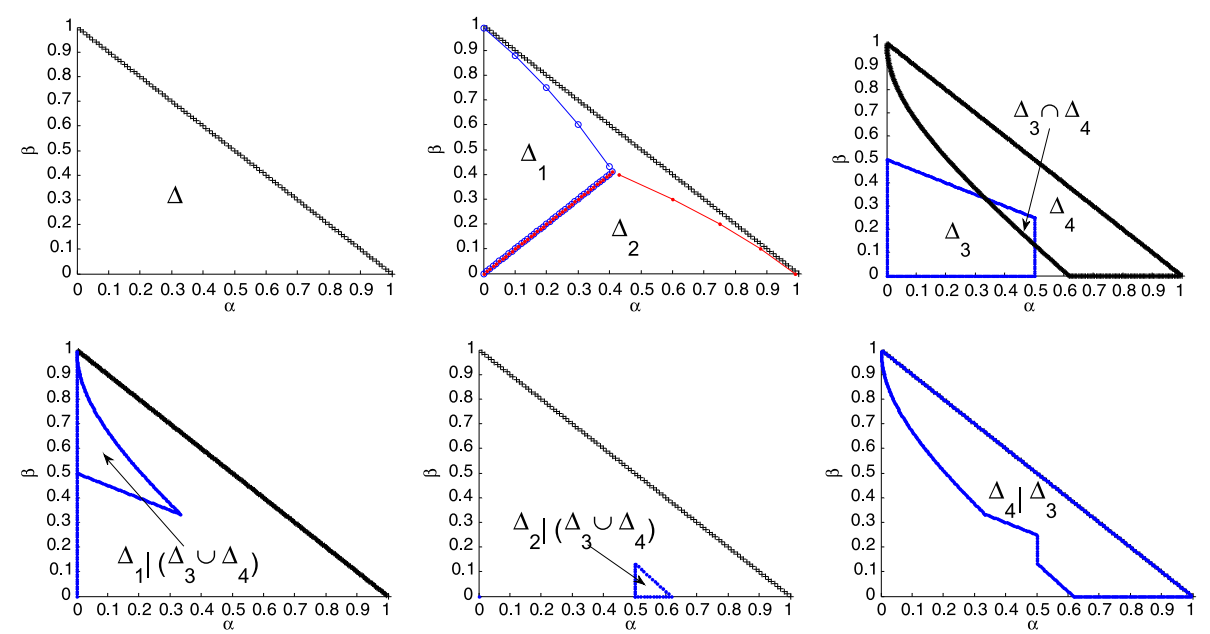

Figure 1 The sets $\Delta$ and $\Delta_{i}, i=1,2,3,4$. 
of them do not imply each other. In this context, note, for instance, that the simple scalar single-valued sequence $x_{n+1}=a x_{n} ; \forall n \in \mathbf{N}_{0}=\mathbf{N} \cup\{0\}$, with initial condition $x_{0} \in \mathbf{R}$, is a strict contraction if $|a|<1$. However, it is not a Kannan contraction for all $|a|<1$. This is easily seen as follows. Check the Kannan condition $d(T x, T y) \leq \alpha(d(x, T x)+d(y, T y))$ for the self-mapping $T$ on $\mathbf{R}$ defining the sequence solution and $\alpha \in[0,1 / 2)$, for instance, for points $x=x_{n}, x_{n+1}=T x, x_{n+2}=T^{2} x=y$ and $x_{n+3}=T^{3} x=T y$ for any $n \in \mathbf{N}_{0}$. Then the Kannan contractive test is subject to $\frac{1}{2}>\alpha \geq \frac{|a|}{1+a^{2}}$, which is not fulfilled for given nonzero sufficiently small values of $1>|a|>0$ and any real $\alpha \in[0,1 / 2)$. It is possible also to check in a similar way a failure of the generalized Kannan-extended contractive condition $d(T x, T y) \leq \alpha d(x, T x)+\beta d(y, T y)$ with $0 \leq \beta<1-\alpha, \alpha \in[0,1)$ for given nonzero sufficiently small values of $1>|a|>0$.

In the current approach, a combination of distinct contractive conditions for the $(\alpha, \beta)$ pairs of values belonging to some relevant sets constructed from the subsets $\Delta_{i} ; i=1,2,3,4$ of $\Delta$ is itself combined with the two point-to-point possibilities of combinations of the comparisons $M_{2}(x, y, \alpha, \beta)>($ or $\leq) M_{1}(x, y, K)$ for each $(x, y) \in A \times B \cup B \times A$. The various constraints are used to prove the convergence of the iterated sequences constructed with cyclic self-mappings $T: A \cup B \rightarrow A \cup B$ to best proximity points. On the other hand, the use of $\omega D$ in the contractive condition, instead of the distance in-between subsets, allows via the choice of some real constant $\omega>1$ to deal with problems where the achievement of limits of sequences at best proximity points is not of particular interest but just their limits superior belonging to certain subsets of the relevant sets $A_{i} \subset X ; i \in \bar{p}$ containing the best proximity points. In this case, the permanence of the relevant sequences after a finite time in subsets of the sets $A_{i} \subset X ; i \in \bar{p}$ after a finite number of steps is guaranteed. The standard analysis can be used for the particular case $\omega=1$. The performed study in the manuscript seems to be also promising for its extension to the study of single-valued and multivalued proximal contraction mappings in-between subsets of metric spaces because of the close formal relation between cyclic self-mappings and proximal mappings. See, for instance, [40] and references therein.

\section{Main results}

The first main result follows.

Theorem 2.1 Let $(X, d)$ be a complete metric space, and let $T: A \cup B \rightarrow A \cup B$ be, in general, a multivalued cyclic self-mapping, where $A, B \subset X$ are nonempty, closed and subject to the contractive constraint

$$
[\varphi(x, y, K, \alpha, \beta) d(x, T x) \leq d(x, y)] \quad \Rightarrow \quad[H(T x, T y) \leq M(x, y, K, \alpha, \beta)+\omega D]
$$

subject to (1.1)-(1.4), for some $\omega \in \mathbf{R}_{+}, K \in[0,1)$ and $(\alpha, \beta) \in \Delta ; \forall(x, y) \in(A \cup B) \times(B \cup A)$. Assume also that

$$
K_{1}=\max \left(K, \frac{\beta}{1-\alpha}, \frac{\alpha}{1-\beta}\right) \in[0,1), \quad K_{2}=\max \left(\frac{1}{1-\alpha}, \frac{1}{1-\beta}\right) \in[1, \infty) .
$$

Then the following properties hold:

(i) There is a sequence $\left\{x_{n}\right\}$ in $A \cup B$ satisfying $x_{i+1} \in T x_{i}, i \in \mathbf{N}$ such that

$$
D \leq d\left(x_{n+1}, x_{n}\right)<\infty ; \quad D \leq \limsup _{n \rightarrow \infty} d\left(x_{n+1}, x_{n}\right) \leq \frac{\omega K_{2} D}{1-K_{1}} .
$$


If $A$ and $B$ are bounded sets which intersect, then $\sum_{n=1}^{\infty} d\left(x_{n+1}, x_{n}\right)<\infty$ and $\left\{x_{n}\right\}$ is a Cauchy sequence having its limit in $A \cap B$, with $x_{n+1} \in T x_{n} ; n \in \mathbf{N}$ for any given $x_{1} \in A \cup B$. If $A$ and $B$ are not bounded, then the above property still holds if $d\left(x_{1}, x_{2}\right)<\infty$. Furthermore, $\lim _{n \rightarrow \infty} d\left(x_{n+1}, x_{n}\right)=D$ exists if $\omega=\frac{1-K_{1}}{K_{2}}$ for any given $x_{1} \in A \cup B$ with the sequence $\left\{x_{n}\right\}$ being constructed in such a way that $x_{n+1} \in T x_{n}$.

If $x_{1} \in A$, then the sequence of sets $\left\{T x_{2 n+1}\right\} \subset T(B) \subseteq A$ converges to a subset $\left\{z_{A}\right\}$ of best proximity points in $A$ (in the sense that any point $z_{2 n+1}\left(\in\left\{T x_{2 n}\right\}\right) \rightarrow z^{0} \in\left\{z_{A}\right\}$ as $n \rightarrow \infty$ ) and the sequence of sets $\left\{T x_{2 n}\right\} \subset T(A) \subseteq B$ converges to a subset $\left\{z_{B}\right\} \subset T\left\{z_{A}\right\} \subset T(A) \subset B$ of best proximity points in $B$ with $\left\{z_{A}\right\} \subset T\left\{z_{B}\right\} \subset T(B) \subset A$.

If $D=0$, i.e., if $A \cap B \neq \emptyset$, then $\lim _{n \rightarrow \infty} \sup _{m>n} d\left(x_{m}, x_{n}\right)=\lim _{n \rightarrow \infty} d\left(x_{n+1}, x_{n}\right)=0$, and any sequence $\left\{x_{n}\right\}$ being iteratively generated as $x_{n+1} \in T x_{n}$, for any $x_{1}=x \in A \cup B$, is a Cauchy sequence which converges to a fixed point $z \in T z \cap(A \cap B)$ of $T: A \cup B \rightarrow A \cup B$.

(ii) Assume that $A \cap B \neq \emptyset$, that $A$ and $B$ are convex, and that $z_{i} \in T z_{i} ; i \in \bar{N}=\{1,2, \ldots, N\}$ are fixed points of $T: A \cup B \rightarrow A \cup B$. Then $z_{i}=z_{j} \subset A \cap B$ and $T z_{i} \equiv T z_{j} \subset A \cap B ; \forall i, j$ $(\neq i) \in \bar{N}=\{1,2, \ldots, N\}$, that is, the image sets of any fixed points are identical.

(iii) Consider a uniformly convex Banach space $(X,\|\|)$, so that $(X, d)$ is a complete metric space for the norm-induced metric $d: X \times X \rightarrow \mathbf{R}_{0_{+}}$, and let $A$ and $B$ be nonempty, disjoint, convex and closed subsets of $X$ with $T: A \cup B \rightarrow A \cup B$ satisfying the contractive conditions (2.1)-(2.2) with $\omega=\frac{1-K_{1}}{K_{2}}$.

Then a sequence $\left\{x_{2 n}\right\}$ built so that $x_{2 n} \in T x_{2 n-1}$ with $x_{2 n-1} \in T x_{2 n-2}$ is a Cauchy sequence in $A$ if $x_{1} \in A$ and $a$ Cauchy sequence in $B$ if $x_{1} \in B$ so that $\lim _{n \rightarrow \infty} d\left(x_{2 n+3}, x_{2 n+1}\right)=$ $\lim _{n \rightarrow \infty} d\left(x_{2 n+2}, x_{2 n}\right)=0 ; \forall x_{1} \in A \cup B$, and $\lim _{n \rightarrow \infty} d\left(x_{2 n+2}, x_{2 n+1}\right)=\lim _{n \rightarrow \infty} d\left(x_{2 n+1}, x_{2 n}\right)=$ $D ; \forall x_{1} \in A \cup B$. If $x_{1} \in A$ and $x_{2} \in T x_{1} \subset T(A) \subset B$, then the sequences of sets $\left\{T^{2 n} x_{1}\right\} \equiv$ $\left\{T\left(T^{2 n-1} x_{1}\right)\right\}$ and $\left\{T^{2 n+1} x_{1}\right\}$ converge to unique best proximity points $z_{A} \in T z_{B}$ and $z_{B} \in T z_{A}$ in $A$ and $B$, respectively.

Proof The proof is organized by firstly splitting it into two parts, namely, the situations: (a) $M_{2}$ defined in (1.2a), or (b) $M_{1}$, defined in (1.2b), gives the maximum for $M$, defined in (1.1); and then in five distinct cases concerning (1.3), subject to (1.4), as follows.

(a) Assume that $M_{2}(x, y, \alpha, \beta)<M_{1}(x, y, K)$. Take, with no loss in generality, $x \in A$ and $y \in T x$ and note that $\varphi(x, y, K, \alpha, \beta) d(x, T x) \leq d(x, T x) \leq d(x, y)$ since $\varphi(x, y, K, \alpha, \beta) \in(0,1]$, which implies that $\varphi(x, y, K, \alpha, \beta) d(x, T x) \leq d(x, T x)$, and since $y \in T x$, then it follows that $d(x, T x) \leq d(x, y)$. Since $M_{2}(x, y, \alpha, \beta)<M_{1}(x, y, K)$, then one gets from the definition of Hausdorff metric (1.5) and the contractive condition (2.1), which holds for any $x, y \in A \cup B$, that for some $y \in T x$,

$$
\begin{aligned}
d(y, T y) & \leq H(T x, T y) \leq M(x, y, K, \alpha, \beta)+\omega D=M_{1}(x, y, K)+\omega D \\
& =K \max \left\{d(x, y), d(x, T x), d(y, T y), \frac{1}{2}(d(x, T y)+d(y, T x))\right\}+\omega D \\
& =K \max \left\{d(x, y), d(y, T y), \frac{d(x, T y)}{2}\right\}+\omega D \\
& =K \max \left\{d(x, y), \frac{d(x, T y)}{2}\right\}+\omega D
\end{aligned}
$$

since $K \in[0,1), d(x, T x) \leq d(x, y)$ and $d(y, T x)=0 ; \forall y \in T x$. Also, since $\beta \in[0,1)$ and $\varphi(y, x, K, \alpha, \beta) d(y, T y) \leq d(y, T y) \leq d(x, y)$, then $d(y, T y) \leq \frac{1}{1-\beta}(d(x, y)+\omega D)$. Thus, there is 
$y \in T x$ such that $d(y, T y) \leq \frac{1}{1-\beta}(d(x, T x)+\omega D)$ so that

$$
\begin{aligned}
d(y, T y) & \leq \min \left(\frac{\alpha}{1-\beta} d(x, T x), K \max \left\{d(x, y), \frac{d(x, T y)}{2}\right\}\right)+\frac{\omega D}{1-\beta} \\
& \leq \min \left(\frac{\alpha}{1-\beta} d(x, T x), K \max \left\{d(x, y), \frac{d(x, y)+d(y, T y)}{2}\right\}\right)+\frac{\omega D}{1-\beta} \\
& \leq \min \left(\frac{\alpha}{1-\beta} d(x, T x), K \max \left\{d(x, y), \frac{d(y, T y)}{2}\right\}\right)+\frac{\omega D}{1-\beta} \\
& \leq \min \left(\frac{\alpha}{1-\beta} d(x, T x), K d(x, y)\right)+\frac{\omega D}{1-\beta} \\
& \leq \max \left(K, \frac{\alpha}{1-\beta}\right) d(x, y)+\frac{\omega D}{1-\beta}
\end{aligned}
$$

since $d(x, T x) \leq d(x, y) ; \forall y \in T x$.

(b) Assume that $M_{2}(x, y, \alpha, \beta) \geq M_{1}(x, y, K)$ so that

$$
d(y, T y) \leq M_{2}(x, y)=\alpha d(x, T x)+\beta d(y, T y)+\omega D
$$

This implies also that $d(y, T y) \leq \frac{\alpha}{1-\beta} d(x, T x)+\frac{\omega D}{1-\beta}$ and again (2.4) holds for $y \in T x$. As a result,

$$
d(y, T y) \leq \max \left(K, \frac{\alpha}{1-\beta}\right) d(x, y)+\frac{\omega D}{1-\beta} ; \quad \forall x \in A, \forall y \in B
$$

By interchanging the roles of the sets $A$ and $B$, one also gets by proceeding in a similar way:

$$
d(x, T x) \leq \max \left(K, \frac{\beta}{1-\alpha}\right) d(x, y)+\frac{\omega D}{1-\alpha} ; \quad \forall x \in A, \forall y \in B
$$

Thus,

$$
\begin{aligned}
d(x, T x) & \leq \max \left(K, \frac{\beta}{1-\alpha}, \frac{\alpha}{1-\beta}\right) d(x, y)+\max \left(\frac{1}{1-\alpha}, \frac{1}{1-\beta}\right) \omega D \\
& =K_{1} d(x, y)+K_{2} \omega D
\end{aligned}
$$

$\forall(x, y) \in(A \cup B) \times[T(A \cup B)]$, where $K_{1}=\max \left(K, \frac{\beta}{1-\alpha}, \frac{\alpha}{1-\beta}\right) \in[0,1)$ and $K_{2}=\max \left(\frac{1}{1-\alpha}, \frac{1}{1-\beta}\right)$. Note that since $T: A \cup B \rightarrow A \cup B$ is cyclic, then $y, T x \in B$ if $x \in A$ and conversely.

Now, construct a sequence $\left\{x_{n}\right\}$ in $A \cup B$ as follows: $x=x_{1} \in A, x_{2} \in T x_{1} \subset T(A) \subset$ $B, \ldots, x_{2 n} \in T x_{2 n-1} \subset T(A) \subset B, \ldots, x_{2 n+1} \in T x_{2 n} \subset T(B) \subset A$ which satisfies

$$
\begin{aligned}
D & \leq d\left(x_{n+1}, x_{n}\right) \leq K_{1} d\left(x_{n}, x_{n-1}\right)+\omega K_{2} D \\
& \leq K_{1}^{n-1} d\left(x_{2}, x_{1}\right)+\left(\sum_{i=0}^{n-2} K_{1}^{i}\right) K_{2} \omega D ; \quad n \in \mathbf{N} \\
& \leq K_{1}^{n-1} d\left(x_{2}, x_{1}\right)+\frac{1-K_{1}^{n-1}}{1-K_{1}} K_{2} \omega D<\infty ; \quad n \in \mathbf{N} .
\end{aligned}
$$


Then $D \leq \lim \sup _{n \rightarrow \infty} d\left(x_{n+1}, x_{n}\right) \leq \frac{K_{2} \omega D}{1-K_{1}}$. On the other hand,

$$
\begin{aligned}
\sum_{n=1}^{j} d\left(x_{n+1}, x_{n}\right) & \leq \sum_{n=1}^{j}\left(K_{1}^{n-1} d\left(x_{2}, x_{1}\right)+\frac{1-K_{1}^{n-1}}{1-K_{1}} K_{2} \omega D\right) \\
& \leq \frac{1}{1-K_{1}}\left[\left(1-K_{1}^{j}\right) d\left(x_{2}, x_{1}\right)+\left(\sum_{n=1}^{j}\left(1-K_{1}^{n-1}\right)\right) K_{2} \omega D\right] ; \quad n \in \mathbf{N}
\end{aligned}
$$

so that

$$
\sum_{n=1}^{\infty} d\left(x_{n+1}, x_{n}\right) \leq \frac{1}{1-K_{1}}\left[d\left(x_{2}, x_{1}\right)+\left(\sum_{n=1}^{\infty}\left(1-K_{1}^{n-1}\right)\right) K_{2} \omega D\right] ; \quad n \in \mathbf{N}
$$

and we conclude that $\left\{x_{n}\right\}$ is a Cauchy sequence if $D=0$ (i.e., if $A$ and $B$ intersect provided that they are bounded or simply if $\left.d\left(x_{2}, x_{1}\right)<\infty\right)$ since $\lim _{n \rightarrow \infty} \sup _{m>n} d\left(x_{m}, x_{n}\right)=$ $\lim _{n \rightarrow \infty} d\left(x_{n+1}, x_{n}\right)=0$, which has a limit $z$ in $X$, since $(X, d)$ is complete, which is also in $A \cap B$ which is nonempty and closed since $A$ and $B$ are both nonempty and closed since $T(A) \subseteq B$ and $T(B) \subseteq A$. On the other hand, for any distance $D \geq 0$ between $A$ and $B$,

$$
\begin{aligned}
D & \leq d\left(x_{2 n+3}, x_{2 n+2}\right) \leq K_{1}^{2 n+1} d\left(x_{2}, x_{1}\right)+\left(\sum_{i=0}^{2 n} K_{1}^{i}\right) \omega D \\
& \leq K_{1}^{2 n+1} d\left(x_{2}, x_{1}\right)+\frac{1-K_{1}^{2 n+1}}{1-K_{1}} K_{2} \omega D ; \quad n \in \mathbf{N}, \\
D & \leq d\left(x_{2 n+2}, x_{2 n+1}\right) \leq K_{1}^{2 n} d\left(x_{2}, x_{1}\right)+\left(\sum_{i=0}^{2 n-1} K_{1}^{i}\right) \omega D \\
& \leq K_{1}^{2 n} d\left(x_{2}, x_{1}\right)+\frac{1-K_{1}^{2 n}}{1-K_{1}} K_{2} \omega D ; \quad n \in \mathbf{N}, \\
0 & \leq d\left(x_{2 n+3}, x_{2 n+1}\right) \leq K_{1} d\left(x_{2 n+2}, x_{2 n}\right)+\omega D \leq K_{1}^{2} d\left(x_{2 n+1}, x_{2 n-1}\right)+\left(1+K_{1}\right) K_{2} \omega D \\
& \leq K_{1}^{3} d\left(x_{2 n}, x_{2 n-2}\right)+\left(1+K_{1}+K_{1}^{2}\right) \omega D \leq K_{1}^{4} d\left(x_{2 n-1}, x_{2 n-3}\right)+\left(1+K_{1}+K_{1}^{2}+K_{1}^{3}\right) K_{2} \omega D \\
& \leq K_{1}^{2 n} d\left(x_{3}, x_{1}\right)+\left(\sum_{i=0}^{2 n-1} K_{1}^{i}\right) \omega D \leq K_{1}^{2 n} d\left(x_{3}, x_{1}\right)+\frac{1-K_{1}^{2 n}}{1-K_{1}} K_{2} \omega D ; \quad n \in \mathbf{N} .
\end{aligned}
$$

Note that the sequences $\left\{d\left(x_{n}, x_{n+1}\right)\right\}$ and $\left\{d\left(x_{n}, x_{n+2}\right)\right\}$ are bounded if $x_{1}$ and $x_{2} \in T x_{1}$ are such that $d\left(x_{1}, x_{2}\right)<\infty$, which is always guaranteed if $A$ and $B$ are bounded. If $\omega=\frac{1-K_{1}}{K_{2}}$, then one gets from the above relations that

$$
\exists \lim _{n \rightarrow \infty} d\left(x_{2 n+3}, x_{2 n+2}\right)=\lim _{n \rightarrow \infty} d\left(x_{2 n+2}, x_{2 n+1}\right)=D,
$$

where $x_{2 n+1} \in T x_{2 n} \subset A, x_{2 n+2} \in T x_{2 n+1} \subset B$ and $x_{2 n+3} \in T x_{2 n+2} \subset A$. Thus, any sequences of sets $\left\{x_{2 n+1}\right\}$ and $\left\{x_{2 n}\right\}$ contain the best proximity points of $A$ and $B$, respectively, if $x_{1} \in A$ and, conversely, of $B$ and $A$ if $x_{1} \in B$ and converge to them. This follows by contradiction since, if not, for each $k \in \mathbf{N}$, there is some $\varepsilon=\varepsilon(k) \in \mathbf{R}_{+}$, some subsequence $\left\{n_{k j}\right\}_{j \in \mathbf{N}}$ of natural numbers with $n_{k m}>n_{k j}>k$ for $m>j$, and some related subsequences of real numbers $\left\{x_{2 n_{k j}+1}\right\}$ and $\left\{x_{2 n_{k j}}\right\}$ such that $d\left(x_{2 n_{k j}+2}, x_{2 n_{k j}+1}\right) \geq D+\varepsilon$ so that $d\left(x_{2 n_{k}+2}, x_{2 n_{k}+1}\right) \rightarrow D$ as $n_{k} \rightarrow \infty$ is impossible. 
Now, assume $D=0$ and consider separately the various cases in (1.3)-(1.4), by using the contractive condition (2.1), subject to (1.1)-(1.4), to prove that there is $z \in T z$ in $A \cap B$ to which all sequences converge by using $D=0 \Rightarrow \lim _{n \rightarrow \infty} \sup _{m>n} d\left(x_{m}, x_{n}\right)=$ $\lim _{n \rightarrow \infty} d\left(x_{n+1}, x_{n}\right)=0 \Rightarrow\left\{x_{n}\right\} \rightarrow z \in A \cap B$ with $\left\{x_{n}\right\}$ being a Cauchy sequence since $(X, d)$ is complete and $A$ and $B$ are nonempty and closed.

Case 1: $\varphi(x, y, K, \alpha, \beta)=1,\left(\left[(\alpha, \beta) \in \Delta_{1}\right] \vee\left[(\alpha, \beta) \in \Delta_{2}\right]\right) \wedge\left(M(z, z, K, \alpha, \beta)=M_{2}(z, z\right.$, $\left.\alpha, \beta)>M_{1}(z, z, K)\right)$.

Then $d(z, T z)=\varphi(z, z, K, \alpha, \beta) d(z, T z) \leq(\alpha+\beta) d(z, T z) \leq\left(1-\alpha^{2}\right) d(z, T z)$ if $(\alpha, \beta) \in \Delta_{1}$. Thus, the contradiction $d(z, T z)<d(z, T z)$ holds if $(\alpha, \beta) \in \Delta_{1}, \alpha \neq 0$ and $z \notin T z$. Hence, $z \in T z$ if $(\alpha, \beta) \in \Delta_{1}$ with $\alpha \neq 0$ since $T z$ is closed. If $\alpha=0$, then $0 \leq \beta<1$ so that $d(z, T z) \leq$ $\beta d(z, T z)<d(z, T z)$ if $z \notin T z$. Hence, $z \in T z$ if $\alpha=0$ and $(0, \beta) \in \Delta_{1}$. The proof that $z \in T z$ if $(\alpha, \beta) \in \Delta_{2}$ is similar since $(\alpha, \beta) \in \Delta_{2} \Leftrightarrow(\beta, \alpha) \in \Delta_{1}$ from the definitions of the sets $\Delta_{1}$ and $\Delta_{2}$, and the fact that distances have the symmetry property.

Case 2: $\varphi(z, z, K, \alpha, \beta)=1-\beta,\left(\left[(\alpha, \beta) \in \Delta_{3}\right]\right) \wedge\left(M(z, z, K, \alpha, \beta)=M_{2}(z, z, \alpha, \beta)>M_{1}(z\right.$, $z, K))$.

Then the contractive condition becomes $(1-\beta) d(z, T z)=\varphi(x, y, K, \alpha, \beta) d(z, T z) \leq(\alpha+$ $\beta) d(z, T z)$. Then either $z \in T z$ or $z \notin T z$ and $1<\alpha+2 \beta$ with $(\alpha, \beta) \in \Delta_{3}$. But the second possibility is impossible since $\Delta_{3}=\left\{(\alpha, \beta) \in \Delta: \alpha \in(0,1 / 2), \frac{1-\alpha}{2} \geq \beta\right\}$ so that $1 \geq \alpha+2 \beta$. Hence, $z \in T z$ since $T z$ is closed.

Case 3: $\varphi(z, z, K, \alpha, \beta)=\frac{1-\beta}{1-\beta+\alpha},\left((\alpha, \beta) \in \Delta_{4}\right) \wedge\left(M(z, z, K, \alpha, \beta)=M_{2}(z, z, \alpha, \beta)>M_{1}(z\right.$, $z, K)$ ).

Then $\frac{1-\beta}{1-\beta+\alpha} d(z, T z)=\varphi(z, z, K, \alpha, \beta) d(z, T z) \leq(\alpha+\beta) d(z, T z)$ if $(\alpha, \beta) \in \Delta_{4}$, which implies for $z \notin T z$ if $(\alpha, \beta) \in \Delta_{4}$ that $\frac{1-\beta}{1-\beta+\alpha}>\alpha+\beta$, equivalently, $1>\alpha(1+\alpha)+\beta(2-\beta)$. Since $\Delta_{4}=\{(\alpha, \beta) \in \Delta: \alpha(1+\alpha)+\beta(2-\beta) \geq 1\}, z \notin T z$ with $(\alpha, \beta) \in \Delta_{4}$ is impossible. Hence, $z \in T z$ since $T z$ is closed.

Case 4: $\varphi(x, y, K, \alpha, \beta)=1,(0 \leq K<1 / 2) \wedge\left(M_{2}(z, z, \alpha, \beta) \leq M(z, z, K, \alpha, \beta)=M_{1}(z, z, K)\right)$.

Then $d(z, T z)=\varphi(z, z, K, \alpha, \beta) d(z, T z) \leq K \max \left\{d(z, z), d(z, T z), \frac{d(z, T z)}{2}\right\}=K d(z, T z)<d(z$, $T z$ ), which is a contradiction for any $z \notin T z$. Hence, $z \in T z$ since $T z$ is closed.

Case 5: $\varphi(x, y, K, \alpha, \beta)=1-K,(1 / 2 \leq K<1) \wedge\left(M_{2}(z, z, \alpha, \beta) \leq M(z, z, K, \alpha, \beta)=M_{1}(z\right.$, $z, K))$.

Then

$$
\begin{aligned}
& (1-K) d(z, T z)=\varphi(z, z, K, \alpha, \beta) d(z, T z) \leq d(z, T z) \\
& \Rightarrow \quad d(z, T z) \leq K \max \left\{d(z, z), d(z, T z), \frac{d(z, T z)}{2}\right\}=K d(z, T z)<d(z, T z),
\end{aligned}
$$

which is a contradiction if $z \notin T z$. Hence, $z \in T z$ since $T z$ is closed. A combined result of Cases 1-5 is that $D=0 \Rightarrow\left\{x_{n}\right\} \rightarrow z \in T z \cap(A \cap B)$ for any $x_{1} \in A \cup B$. Now, assume again that $A \cap B \neq \emptyset$ and that there are two distinct fixed points $z_{x}\left(\neq z_{y} \in T z_{y}\right) \in T z_{x}$ necessary located in $A \cap B$ to which the sequences $\left\{x_{n}\right\}$ and $\left\{y_{n}\right\}$ converge to $z \in A \cap B$ and $q(\neq z) \in A \cap B$, respectively, where $x_{n+1} \in T x_{n}, y_{n+1} \in T y_{n}$ for $n \in \mathbf{N}$, where $x_{1}, y_{1}\left(\neq x_{1}\right) \in A \cup B$. Assume also that $T z \neq T q$. One gets from the contractive condition (2.1), subject to (1.1)-(1.4), that

$$
\begin{aligned}
\max (d(z, T q), d(q, T z)) & \leq \max \left(\sup _{x \in T z} d(x, T q), \sup _{y \in T q} d(y, T z)\right) \\
& \leq K \max (d(z, q), 1 / 2(d(z, T q)+d(q, T z)))=K d(z, q)<d(z, q) .
\end{aligned}
$$


Thus, construct sequences $z_{n+1} \in T z_{n}, q_{n+1} \in T q_{n}$ with $z_{1}=z$ and $q_{1}=q$ such that $d\left(z, q_{n+1}\right)<d\left(z, q_{n}\right)$ and $d\left(q, z_{n+1}\right)<d\left(q, z_{n}\right)$ for $n \in \mathbf{N}$. Since $z, q \in A \cap B$ which is nonempty, closed and convex, for any given $\varepsilon \in \mathbf{R}_{+}$, there is $n_{0}=n_{0}(\varepsilon)$ such that $x_{n}$ and $q_{n}$ are in $A \cap B$ for $n \geq n_{0}$. Then $q_{n} \rightarrow \hat{z}(\in T z)$ and $z_{n} \rightarrow \hat{q}(\in T q)$ as $n \rightarrow \infty$ with $z \in T z \cap A \cap B$ and $q \in T q \cap A \cap B$. Hence, $T z \equiv T q$ in $A \cap B$ contradicting the hypothesis that such sets are distinct. Properties (i)-(ii) have been proven.

Property (iii) is proven by using, in addition, [35, Lemma 3.8], one gets

$$
\exists \lim _{n \rightarrow \infty} d\left(x_{2 n+3}, x_{2 n+2}\right)=\lim _{n \rightarrow \infty} d\left(x_{2 n+2}, x_{2 n+1}\right)=D \quad \Rightarrow \quad \lim _{n \rightarrow \infty} d\left(x_{2 n+3}, x_{2 n+1}\right)=0
$$

for any sequence $\left\{x_{n}\right\}$ with $x_{1} \in A \cup B$ and $x_{n+1} \in T x_{n}$ since $(X, d)$ is a uniformly convex Banach space, $A$ and $B$ are nonempty and disjoint closed subsets of $X$ and $A$ is convex. Note that Lemma 3.8 of [35] and its given proof remain fully valid for multivalued cyclic self-maps since only metric properties were used in its proof. It turns out that $\left\{x_{2 n+1}\right\}$ is a Cauchy sequence, then bounded, with a limit $z_{A}$ in $A$, which is also a best proximity point of $T: A \cup B \rightarrow A \cup B$ in $A$ since

$$
\begin{aligned}
\lim _{n \rightarrow \infty} d\left(x_{2 n+2}, x_{2 n+1}\right) & =\lim _{n \rightarrow \infty} d\left(x_{2 n+2}, z_{A}\right)=D \leq \lim _{n \rightarrow \infty} d\left(x_{2 n+2}, x_{2 n}\right)+\lim _{n \rightarrow \infty} d\left(x_{2 n}, z_{A}\right) \\
& =\lim _{n \rightarrow \infty} d\left(x_{2 n}, z_{A}\right) \leq D
\end{aligned}
$$

and then $\left\{x_{2 n}\right\}$ converges to some point $z_{B} \in T z_{A} \subset B$, which is also a best proximity point in $B$ (then $z_{B} \in T z_{A}$ and $T z_{A} \subset B$ ), since $(X, d)$ is a uniformly convex Banach space and $A$ and $B$ are nonempty closed and convex subsets of $X$. In the same way, $z_{A} \in T z_{B} \subset A$. Also, $\left\{x_{2 n}\right\}$ and $\left\{x_{2 n+1}\right\}$ are bounded sequences since $\left\{x_{2 n}\right\}$ is bounded and $D<\infty$. Also, if $x_{1} \in B$ and $B$ is convex, then the above result holds with $x_{2 n+1} \in T x_{2 n} \subset B, x_{2 n+2} \in T x_{2 n+1} \subset A$ and $x_{2 n+3} \in T x_{2 n+2} \subset B$. Now, for $D>0$, the reformulated five cases in the proof of Property (i) would lead to contradictions $D=d\left(z_{A}, z_{B}\right)<D \neq 0$ if $z_{A} \notin T z_{B}$ or if $z_{B} \notin T z_{A}$. From Proposition 3.2 of [35], there are $z_{A} \in T z_{B}$ and $z_{B} \in T z_{A}$ such that $D=d\left(z_{A}, T z_{A}\right)=d\left(z_{B}, T z_{B}\right)$ since $T: A \cup B \rightarrow A \cup B$ is cyclic satisfying the contractive conditions (2.1)-(2.2), where $A$ and $B$ are nonempty and closed subsets of a complete metric space $(X, d)$, with convergent subsequences $\left\{x_{2 n+1}\right\}$ and $\left\{x_{2 n}\right\}$ in both $A$ and $B$, respectively, for any $x=x_{1} \in A$ and in $B$ and $A$, respectively, for any given $x=y_{1} \in B$. Assume that some given sequence $\left\{x_{2 n+1}\right\}$ in $A$ is generated from some given $x_{1} \in A$ with $x_{2 n+1} \in T x_{2 n}$, which converges to the best proximity point $z_{A} \in A \cap T z_{B}$ in $A$ of $T: A \cup B \rightarrow A \cup B$. Assume also that there is some sequence $\left\{y_{2 n}\right\}$, distinct from $\left\{x_{2 n}\right\}$, in $A$ generated from $y_{1}\left(\neq x_{1}\right) \in A$ with $y_{2 n+1} \in T y_{2 n}$ which converges to $z_{A 1} \in A$, where $z_{B} \in B \cap T z_{A}$ is a best proximity point in $B$ of $T: A \cup B \rightarrow A \cup B$. Consider the complete metric space $(X, d)$ obtained by using the norm-induced metric in the Banach space $(X,\|\|)$ so that both spaces can be mutually identified to each other. Since $d(x, y) \geq D$ for any $x \in A$ and $y \in B$, it follows that $D=d\left(z_{B}, z_{A}\right)=d\left(z_{A}, T z_{A}\right)=d\left(z_{B}, T z_{B}\right)<d\left(z_{A 1}, T z_{A}\right)$ if $z_{A 1} \in A \cap \overline{T z_{B}}$, where $z_{A}$ and $z_{B}$ are best proximity points of $T: A \cup B \rightarrow A \cup B$ in $A$ and $B$ and $\overline{T z_{B}}$ is the closure of $T z_{B}$. Hence, $z_{A}, z_{A 1} \in T z_{B}$ and $z_{B} \in T z_{A}$ and then any sequence converges to best proximity points.

It is now proven by contradiction that the best proximity points in $A$ and $B$ are unique. Assume that $x, y \in A$ are two distinct best proximity points of $T: A \cup B \rightarrow A \cup B$ in $A$. Then there are $z_{x} \in(T x \cap B) \subset B, z_{x} \in(T y \cap B) \subset B, z_{x}^{\prime} \in\left(T z_{x} \cap A\right) \subset\left(T^{2} x \cap A\right) \subset A$ and 


$$
\begin{aligned}
& z_{y}^{\prime} \in\left(T z_{y} \cap A\right) \subset\left(T^{2} y \cap A\right) \subset A \text { so that, one gets } \\
& \qquad D=\left\|x-z_{x}\right\|=\left\|z_{x}^{\prime}-z_{x}\right\|=\left\|y-z_{y}\right\|=\left\|z_{y}^{\prime}-z_{y}\right\|,
\end{aligned}
$$

which leads to the contradiction $D>\left\|y-z_{x}\right\|=\left\|z_{x}^{\prime}-z_{x}\right\|=D$, and then $x=y=z_{A} \in A$. Hence Property (iii) has been proven.

A special case of Theorem 2.1 is stated and proven in the subsequent result.

Corollary 2.2 Assume that $(X,\|\|)$ is a uniform Banach space with associate norminduced metric $d: X \times X \rightarrow \mathbf{R}_{0_{+}}$, and let $A$ and $B$ be nonempty closed and convex subsets of $X$. Assume also that $K=0, K_{1}=\max \left(\frac{\beta}{1-\alpha}, \frac{\alpha}{1-\beta}\right) \in[0,1), K_{2}=\max \left(\frac{1}{1-\alpha}, \frac{1}{1-\beta}\right) \in[1, \infty)$ and $\omega=\frac{1-K_{1}}{K_{2}}$ in the contractive condition (2.1). If $\max (\alpha, \beta)<1 / 2$, then there are $z_{1} \in A$ and $z_{2} \in B$ such that $z_{1} \in\left(T^{2} z_{1} \cap T z_{2}\right), z_{2} \in\left(T^{2} z_{2} \cap T z_{1}\right)$, i.e., $z_{1}$ and $z_{2}$ are, respectively, best proximity points of $T: A \cup B \rightarrow A \cup B$ in $A$ and $B$, respectively, and simultaneously, fixed points of $T^{2}: A \cup B \rightarrow A \cup B$, respectively. In addition, if $A \cap B \neq \emptyset$, then $\exists z \in$ Tz is a fixed point of $T: A \cup B \rightarrow A \cup B$. The result also holds if $\max (\alpha, \beta)<1$ (and, in particular, if $\min (\alpha, \beta)=0)$.

Proof Assume, with no loss in generality, that $0 \leq \beta \leq \alpha \in[0,1 / 2)$. Take $u_{1} \in A$ and $u_{2} \in$ $T u_{1}$ by noting that $u_{2} \in T u_{1} \subset T(A) \subset B$ since $T: A \cup B \rightarrow A \cup B$ a multivalued cyclic self-mapping.

Remark 2.3 Note that the particular case $M(T x, T y, K, \alpha, \beta)=M_{2}(T x, T y, \alpha, \beta)$ in the contractive condition (2.1) is useful to investigate multivalued cyclic Kannan self-mappings which are contractive with $\alpha=\beta \in[0,1 / 2)$ and some of their generalizations [33, 34].

The following result follows directly from Theorem 2.1 and Corollary 2.2 without proof.

Corollary 2.4 Assume that $T: A \cup B \rightarrow A \cup B$ is a single-valued cyclic self-mapping where $A$ and $B$ are nonempty closed subsets of $X$ where $(X, d)$ is a complete metric space. Then Theorem 2.1 and Corollary 2.2 still hold mutatis-mutandis for a fixed point $z=T z \in A \cap B$ if $A$ and $B$ are convex and intersect and best proximity points are $z_{A} \in A, z_{B} \in B$ with $z_{A}=T z_{B}=T^{2} z_{A}$, if, in addition $(X,\|\|)$ is a uniformly convex Banach space.

Remark 2.5 The results of this section can be extended mutatis-mutandis to multivalued $s(\geq 2)$-cyclic self-maps $T: \bigcup_{i \in \bar{s}} A_{i} \rightarrow \bigcup_{i \in \bar{s}} A_{i}$, where $\bar{s}=\{1,2, \ldots, s\}, A_{i}(\neq \emptyset) \subset X$, $T\left(A_{i}\right) \subseteq A_{i+1}$ and $A_{s+1} \equiv A_{1}$ with $(X, d)$ being a complete metric space. See $[2,3,36,37]$ and references therein for some background results for single-valued cyclic $s$-self-mappings.

\section{Example of application to time-varying discrete-time dynamic systems}

\subsection{Multi-control discrete-time linear dynamic system}

The problems of stability in differential equations, difference equations and related dynamic systems are closely related to fixed point theory of single-valued functions since stable equilibrium points are fixed points [41-44]. Also, fixed point theory of a class of cyclic self-mappings has been recently applied to differential and difference impulsive equations in a stability context study [44]. On the other hand, some typical applications of multivalued maps can be located in the framework of dynamic programming techniques for 
optimal control of dynamic systems $[26,45]$. Several tentative controls are tested to obtain the one which minimizes a suitable cost function on a certain ahead time-interval. One of them is selected as the optimal one. Đorić and Lazović discussed in [1] a dynamic programming application in the continuous-time domain of contractive multivalued-self maps under the theoretical results of their paper. Switches among distinct parameterizations of a dynamic system and the associate stabilization problem have been discussed in the literature. Also, switching processes among different estimators of unknown systems according to the optimization or suboptimization of some appropriate loss function have been described so as to improve the estimation error. See, for instance, [41-43] and included references. On the other hand, fixed point theory has been shown to be useful to discuss the stability of iterative sequences and, in general, for the analysis of the stability of discrete dynamic systems. See, for instance, [46] and references therein. We now discuss a linear time-varying discrete control problem under several tentative controls at each stage with the purpose of selecting the control sequence which guarantees a prescribed stability degree of the feedback system. The problem is stated in such a way that the tentative state-trajectory solution is formally stated as a multivalued function generating several point-to-point iterated sequences and one of them is being selected. In particular, each current state generates a set of tentative ones at the next sampling time which belongs to the image set of the current sampled state. The convergence to fixed points or to best proximity points, if the trajectory solution sequence has a cyclic nature, describes the convergence either to equilibrium points or to a limit cycle of the solution. This second case occurs when the mapping defining the state-trajectory solution is cyclic and the subsets on whose union such a mapping is defined do not intersect. Consider the discrete timevarying control system:

$$
x_{n+1}=A_{n} x_{n}+B_{n} u_{n} ; \quad n \in \mathbf{N}_{0}=\mathbf{N} \cup\{0\},
$$

where $x_{n} \in \mathbf{R}^{p}$ is the state vector sequence for any $n \in \mathbf{N}_{0}=\mathbf{N} \cup\{0\}$ under some nonzero initial state $x_{0} \in \mathbf{R}^{p}$ and $u_{n}=K_{n} x_{n} \in \mathbf{R}^{q}$ for some $1 \leq q \leq p$ and all $n \in \mathbf{N}_{0}$ is the linear time-varying control where $K_{n} \in S_{K n}=\left\{K_{n}^{1}, K_{n}^{2}, \ldots, K_{n}^{J_{n}}\right\} ; n \in \mathbf{N}_{0}$ is a sequence of control gain matrices in $\mathbf{R}^{p \times q}$ which is chosen from an admissible set $S_{K n}$ of cardinal $J_{n} \leq J<\infty$ values for each $n \in \mathbf{N}_{0}$. System (3.1) is said to be an uncontrolled (or open-loop) system if the control sequence is identically zero [26]. The controlled (or closed-loop) system for any time-varying control being generated by a state-feedback control law under a gain matrix sequence $K_{n} \in S_{K n}$ results to be

$$
x_{n+1}=M_{n} x_{n}=A_{n} x_{n}+B_{n} u_{n}=\left(A_{n}+B_{n} K_{n}\right) x_{n} ; \quad n \in \mathbf{N}_{0},
$$

where $\left\{M_{n}\right\}$ is a sequence of matrices in $\mathbf{R}^{p \times q}$ of closed-loop dynamics. The stabilization via linear state-feedback of (3.2) and its links to fixed point theory via Theorem 2.1 are now discussed. For any sequence of natural numbers $j_{n} \in \mathbf{N}$ with $n \in \mathbf{N}_{0}$, the following relation is obtained from (3.2):

$$
x_{n+j_{n}}-\left(\prod_{i=n}^{n+j_{n-1}}\left[A_{i}\right]\right) x_{0}=C\left(n, j_{n}\right) u\left(n, j_{n}\right) ; \quad n \in \mathbf{N}_{0}
$$


where

$$
\begin{aligned}
& C\left(n, j_{n}\right)=\left[B_{n+j_{n}-1}, A_{n+j_{n}-1} B_{n+j_{n}-2}, \ldots,\left(\prod_{i=j_{n}+1}^{n+j_{n}-1}\left[A_{i}\right]\right) B_{j_{n}}\right] ; \quad n \in \mathbf{N}_{0}, \\
& u\left(n, j_{n}\right)=\left[u_{n+j_{n}-1}, u_{n+j_{n}-2}, \ldots, u_{j_{n}}\right]^{T} ; \quad n \in \mathbf{N}_{0},
\end{aligned}
$$

where the superscript ' $T$ ' denotes matrix transposition. Note that if $j_{n}=p$ for $n \in \mathbf{N}_{0}$, then $C(n, p)$ is the $p \times q p$ controllability matrix of (3.1) on the sequence of samples $\{j\}_{n}^{n+p-1}$. Any prefixed state is reachable in any given prefixed number of samples from a null initial condition by some linear time-invariant state-feedback control in at most $p$ samples if and only if (3.1) is reachable, that is, if

$$
\operatorname{rank} C\left(n, j_{n}\right)=\operatorname{rank}\left(\sum_{i=j_{n}}^{n+j_{n}-1}\left(\prod_{k=i+1}^{n+j_{n}-1}\left[A_{k}\right]\right) B_{i} B_{i}^{T}\left(\prod_{k=i+1}^{n+j_{n}-1}\left[A_{k}\right]\right)^{T}\right)=p
$$

for any sequence of integers $\left\{j_{n}\right\}$ with $j_{n} \geq p, n \in \mathbf{N}_{0}$ with $j_{0}=0$ such that $\left\{j_{n+1}-j_{n}\right\}$ is uniformly bounded. It is controllable to the origin if and only if it is reachable, that is, (3.6) holds and, furthermore, $A_{n}$ are all non-singular for $n \in \mathbf{N}_{0}$. It is well known that if the dynamic system (3.1) is controllable to the origin, then it is also stabilizable in the sense that some linear time-varying state-feedback control sequence $\left\{u_{k}\right\}$ is such that $x_{n} \rightarrow 0$ as $n \rightarrow \infty$ for any $x_{0} \in \mathbf{R}^{n}$. The controllability assumption can be weakened while keeping the stabilizability property as follows.

Proposition 3.1 Assume that (3.1) is stabilizable (which is guaranteed if it is controllable to the origin).

Then the following properties hold:

(i) There is a sequence of control gain matrices $\left\{K_{n}\right\}$ such that all the matrices in the subsequence $\left\{\prod_{i=0}^{j_{n}-1}\left[A_{j_{n}+i}+B_{j_{n}+i} K_{j_{n}+i}\right]\right\}$ are convergent matrices with $j_{n} \in \mathbf{N}$ with $j_{0}=0$ being some existing sequence with $\left\{j_{n+1}-j_{n}\right\}$ being a uniformly bounded sequence for any $n \in \mathbf{N}_{0}$.

(ii) The subsequence $\left\{x_{\sum_{i=0}^{n} j_{i}}\right\}$ of states of the closed-loop system (3.2) converges to zero as $n \rightarrow \infty$. As a result, the sequence $\left\{x_{n}\right\}$ of states of the closed-loop system also converges to zero as $n \rightarrow \infty$.

Proof One gets from (3.2) that if such a sequence $\left\{j_{n}\right\}$ of finite natural numbers exists for $n \in \mathbf{N}_{0}$ with $j_{0}=0$, then

$$
\begin{aligned}
x_{j_{n}+j_{n+1}} & =\left(\prod_{i=0}^{j_{n+1}-1}\left[A_{j_{n}+i}+B_{j_{n}+i} K_{j_{n}+i}\right]\right) x_{j_{n}} \\
& =\prod_{k=0}^{n}\left\{\left(\prod_{i=0}^{j_{k+1}-1}\left[A_{j_{k}+i}+B_{j_{k}+i} K_{j_{k}+i}\right]\right)\right\} x_{0} \rightarrow 0
\end{aligned}
$$

as $n \rightarrow \infty$ for some existing sequence of stabilizing controller gains $\left\{K_{n}\right\}$ since $\left(\prod_{i=0}^{j_{n+1}-1}\left[A_{j_{n}+i}+B_{j_{n}+i} K_{j_{n}+i}\right]\right)$, and then $\prod_{k=0}^{n}\left\{\left(\prod_{i=0}^{j_{k+1}-1}\left[A_{j_{k}+i}+B_{j_{k}+i} K_{j_{k}+i}\right]\right)\right\}$ are all convergent matrices, i.e., with all their eigenvalues being of modulus less than one. Note that, since system (3.1) is stabilizable, then such a sequence of nonnegative integers $\left\{j_{n}\right\}$ always exists 
since it exists with $j_{n}=1$ for all $n \in \mathbf{N}_{0}$. Now, it follows from (3.7) for any vector-induced matrix norm that

$$
\begin{aligned}
\left\|x_{j}\right\| & =\left\|\left(\prod_{k=\sum_{i=0}^{n} j_{i}}^{j-1}\left[A_{k}+B_{k} K_{k}\right]\right) x_{\sum_{i=0}^{n} j_{i}}\right\| \\
& \leq\left\|\left(\prod_{k=\sum_{i=0}^{n} j_{i}}^{j-1}\left[A_{k}+B_{k} K_{k}\right]\right)\right\|\left\|\left(\prod_{k=0}^{n}\left\{\left(\prod_{i=0}^{j_{k+1}-1}\left[A_{j_{k}+i}+B_{j_{k}+i} K_{j_{k}+i}\right]\right)\right\}\right) x_{0}\right\| \rightarrow 0
\end{aligned}
$$

as $n \rightarrow \infty$ for any integer $j \in\left(\sum_{i=0}^{n} j_{i}, \sum_{i=0}^{n+1} j_{i}\right)$ since $\|\left(\prod_{k=0}^{n}\left\{\left(\prod_{i=0}^{j_{k+1}-1}\left[A_{j_{k}+i}+B_{j_{k}+i} K_{j_{k}+i}\right]\right)\right\}\right) \times$ $x_{0} \| \rightarrow 0$ as $n \rightarrow \infty$, and

$$
\left\|\left(\prod_{k=\sum_{i=0}^{n} j_{i}}^{j-1}\left[A_{k}+B_{k} K_{k}\right]\right)\right\| \leq\left\|\left(\prod_{k=\sum_{i=0}^{n} j_{i}}^{j_{n+1}-1}\left[A_{k}+B_{k} K_{k}\right]\right)\right\| \leq Q<\infty
$$

since the sequence $\left\{j_{n+1}-j_{n}\right\}$ is uniformly bounded. Thus, $\left\{x_{n}\right\}$ converges to zero for any given $x_{0} \in \mathbf{R}^{n}$.

Proposition 3.1 is linked to Theorem 2.1 of Section 2 in the subsequent result.

\section{Theorem 3.2 The following properties hold:}

(i) Assume that system (3.1) is stabilizable and a linear time-varying feedback control $u_{n}=K_{n} x_{n} \in \mathbf{R}^{q}$ is used where $K_{n} \in S_{K n}=\left\{K_{n}^{1}, K_{n}^{2}, \ldots, K_{n}^{I_{n}}\right\}$ for any $n \in \mathbf{N}_{0}$. Assume also that for each $j \in\left[\sum_{k=0}^{n} j_{k}, \sum_{k=0}^{n+1} j_{k}\right)$ for some sequence of nonnegative integers $\left\{j_{n}\right\}$, such that $\left\{j_{n+1}-j_{n}\right\}$ is uniformly bounded, for any $n \in \mathbf{N}_{0}$ with $j_{0}=0$, there is a controller gain $K_{j}^{k} \in S_{K J_{n}}=\left\{K_{\sum_{k=0}^{n} j_{k}}^{1}, K_{\sum_{k=0}^{n} j_{k}}^{2}, \ldots, K_{\sum_{k=0}^{n} j_{k}}^{J_{n}}\right\}$ for some integer $k \in\left[1, J_{j_{n}}\right]$ with $J_{n} \leq J<\infty$ for all $n \in \mathbf{N}_{0}$ such that any matrix in the subsequence of matrices $\left\{\prod_{i=0}^{j_{n+1}-1}\left[A_{\sum_{k=0}^{n} j_{k}+i}+\right.\right.$ $\left.B_{\sum_{k=0}^{n} j_{k}+i} K_{\sum_{k=0}^{n} j_{k+i}+}^{k}\right]$ is convergent for each $k \in\left[1, J_{j_{n}}\right]$ for some uniformly bounded sequence of samples $\left\{j_{n+1}-j_{n}\right\}$ and some set of upper-bounded positive integer numbers $J_{n}$ for all $n \in \mathbf{N}_{0}$.

(ii) If, in addition, the elements of the subsequence of pairs $\left\{\left(A_{j_{n}}, B_{j_{n}}\right)\right\}$ are all controllable for some sequence of nonnegative integers $\left\{j_{n}\right\}$, with the sequence of natural numbers $\left\{j_{n+1}-\right.$ $\left.j_{n}\right\}$ being uniformly bounded, then the closed-loop system can be exponentially stabilized via time-varying linear control with prescribed stability degree.

Outline of proof Property (i) follows directly from Proposition 3.1. Property (ii) follows since all the pairs $\left(A_{j_{n}}, B_{j_{n}}\right)$ being controllable implies that the matrices of the closed-loop dynamics satisfy at the subsequence $\left\{j_{n}\right\}$ of samples the following matrix relation:

$$
M_{j_{n}}=A_{j_{n}}+B_{j_{n}} K_{j_{n}}=A_{j_{n}}+\sum_{i=1}^{q} B_{j n}^{(i)} K_{j n}^{(i)^{T}} \approx\left[\begin{array}{cc}
0 & I_{p-1} \\
g_{j_{n}}^{T}
\end{array}\right],
$$

where the superscript ' $(i)$ ' stands for the $i$ th row vector of matrix, ' $\approx$ ' stands for matrix similarity, $I_{p-1}$ denotes the $(p-1)$ identity matrix and $g_{j_{n}}^{T}$ denotes some prefixed $p$-real row vector by the appropriate choice of the real controller matrix $K_{j_{n}}$, since $\left(A_{j_{n}}, B_{j_{n}}\right)$ is controllable, towards the achievement of a suitable closed-loop stability degree. Note that the 
closed-loop matrix of dynamics at the $j_{n}$-sample is similar by a similarity transformation to its companion block partitioned form in (3.10). Thus, both matrices have the same characteristic monic polynomial, thus the same characteristic roots which are also the prefixed eigenvalues of the closed-loop dynamics given by (3.10), which can be arbitrarily fixed via $K_{j_{n}}$ such that its non-leading real coefficients are the components of the real row vector $g_{j_{n}}^{T}$. Thus, the sequence of closed-loop matrices can be chosen with the sequence $M_{j_{n}}$ having a stability degree $\rho_{j_{n}} \in(0,1)$ such that the stability degree of $\left(\prod_{i=0}^{j_{n+1}-1}\left[M_{j_{n}+i}\right]\right) M_{j_{n}} \leq \rho \in(0,1)$. This follows since $\left\|\prod_{i=0}^{j_{n+1}-1}\left[M_{j_{n}+i}\right]\right\| \leq P<\infty$ since $\left\{j_{n+1}-j_{n}\right\}$ is uniformly bounded. Thus, the time-varying closed-loop system is exponentially stable with prescribed stability degree $\rho$ and $\left\|x_{j}\right\| \leq P \rho^{n}\left\|x_{0}\right\|$ for any integer $j \in\left[j_{n}, j_{n+1}\right)$ and $n \in \mathbf{N}_{0}$.

The stability degree is defined by the modulus of the dominant eigenvalue of the matrix of dynamics if the dominant eigenvalue is simple and such a number is a strict upperbound of the stability degree, otherwise. At samples which are not in the subsequence $\left\{j_{n}\right\}$, the controller gains may be chosen arbitrarily. The exponential stabilization of the closed-loop system is now related to Theorem 2.1 as follows. Assume that the sequence $\left\{S_{K j_{n}}\right\}$ of sets of matrices $S_{K j_{n}}=\left\{K_{j_{n_{n}}}^{1}, K_{j_{n}}^{2}, \ldots, K_{j_{n}}^{J_{n_{n}}}\right\}$ contains at least a stabilizing matrix such that Theorem 3.2(ii) holds via stabilization with such stabilizing matrices.

Then Theorem 2.1 is applicable to some subset $A=B \subset X \equiv \mathbf{R}^{p}$ being a nonempty bounded set about $0 \in \mathbf{R}^{p}$ such that the initial condition of (3.1) satisfies $x_{0} \in A_{0} \subset A$ of with $D=0$ since $A=B$. Take the distance function equal to the Euclidean norm so that we can consider the complete metric space $(X, d)$ to be identical to the Banach space $(X,\|\|)$. Re-denote the sequence of points in $A$ as the states $x_{j_{0}} \equiv x_{0}\left(\in A_{0}\right) \rightarrow x_{1} \in A$ (the replacement is made following the notation of Theorem 2.1), $x_{j_{1}} \equiv x_{j_{1}}(\in A) \rightarrow x_{2} \in A, x_{j_{2}} \equiv x_{j_{2}}$ $(\in A) \rightarrow x_{3} \in A, \ldots$. If Theorem 3.2(ii) holds, then there is some bounded $A \supset A_{0}$ such that $T: A \rightarrow A$ defined by $\left(T x_{n}=x_{n+1}\right) \rightarrow\left(\left(\prod_{i=0}^{j_{n+1}-1}\left[M_{j_{n}+i}\right]\right) M_{j_{n}} x_{j_{n}}=x_{j_{n+1}}\right)$ for $n \in \mathbf{N}$ is a contractive mapping which defines the state trajectory solution at the points of the sampling subsequence $\left\{j_{n}\right\}$. Take $K_{1}=K=\rho<1$ and $\alpha=\beta=0$ in Theorem 2.1. Note that if the stabilizing matrix is chosen within the sequence of matrices, then $T: A \rightarrow A$ is single-valued. If all the matrices in the sequence $K J_{n}=\left\{K_{\sum_{k=0}^{n} j_{k}}^{1}, K_{\sum_{k=0}^{n} j_{k}}^{2}, \ldots, K_{\sum_{k=0}^{n} j_{k}}^{J_{n}}\right\}$ are tested, then the multivalued map $T: A \rightarrow A$ is defined as $T x_{n}=\left\{x_{n+1}^{1}, \ldots, x_{n+1}^{J_{n}}\right\}$ such that $x_{n}, x_{n+1} \in T x_{n}$ satisfies the Hausdorff particular contractive condition of Theorem 2.1. Also, one of the points of the sequence of sets $\left\{T x_{n}\right\}$ satisfies the point-to-point contractive particular condition of Theorem 2.1, by virtue of such a theorem, according to the constraints

$$
\left\|\left(T_{j_{n+1}}-I\right) x_{j_{n+1}}\right\| \leq\left\|\left(T_{j_{n+1}}-I\right)\right\|\left\|x_{j_{n+1}}\right\| \leq K_{1}\left\|\left(T_{j_{n}}-I\right) x_{j_{n}}\right\| \leq K_{1}^{n}\left\|\left(T_{j_{n}}-I\right) x_{0}\right\|
$$

obtained from the stabilizing control matrix sequence, and, furthermore, $\left\|x_{j_{n}+j}\right\| \leq$ $P K_{1}\left\|x_{j_{n}}\right\|$ for all samples given by the integers $j \in\left[1, j_{n+1}\right)$. Note that the $\ell_{2}$-matrix norm of any real matrix $M$ of any order satisfies $\lambda_{\min }^{1 / 2}\left(M^{T} M\right) \leq\|M\|_{2}=\lambda_{\max }^{1 / 2}\left(M^{T} M\right)$, where $\lambda_{\max }(\cdot)$ and $\lambda_{\min }(\cdot)$ stand, respectively, for the maximum and minimum eigenvalues of the (.)-matrix with all its eigenvalues being real. A weak result is obtained with the particular case $K=\beta=0$ and $K_{1}=\frac{\alpha}{1-\alpha}=\frac{\rho}{1-\rho}<1$ for $0<\rho<1 / 2$ in the contractive condition of Theorem 2.1. In this case, we have a multivalued contractive Kannan self-mapping. In both cases, $0 \in \mathbf{R}^{p}$ is a fixed point of $T: A \rightarrow A$ for any $A \subset X$ which is also a stable equilibrium point of the closed-loop dynamic system. Now assume $p=q=1$, that is, the uncontrolled 
system (3.1) is scalar subject to a scalar control with $A=\{z \geq \varepsilon: z \in \mathbf{R}\} \subseteq \mathbf{R}_{0+}$ and $B=-A$ for some given $\varepsilon \in \mathbf{R}_{0+}$. Take $x_{0} \in A \cup B$, then $\left|x_{0}\right| \geq \varepsilon$, and note that $D=\operatorname{dist}(A, B)=2 \varepsilon$. The tentative controller gains used are $K_{n}^{i}=-\delta_{n}^{i} \operatorname{sgn} x_{n}$ if $x_{n} \neq 0$ and $K_{n}^{i}=0$ if $x_{n}=0$ for the bounded sets of integers $i \in\left[1, J_{n}\right]$ for $n \in \mathbf{N}_{0}$, where the nonnegative real sequences of sets $\left\{\delta_{n}^{i}: 1 \leq i \leq J_{n}\right\}$ are uniformly bounded and contain a strictly decreasing positive real sequence $\left\{\delta_{j_{n}}^{i_{n}}\right\}$ with $i_{n} \in\left[1, J_{j_{n}}\right]$ and some existing difference sequence of integers $\left\{j_{n+1}-j_{n}\right\}$ being uniformly upper-bounded for $n \in \mathbf{N}_{0}$.

The formalism of Section 2 is applicable to bounded sets $A_{0} \subset A$ and $B_{0} \subset B$ with $\max \left(\operatorname{diam} A_{0}, \operatorname{diam} B_{0}\right)=|| x_{0}|-\varepsilon|$. If $\varepsilon=0$, then a particular case of the above result follows for $p=1$. If $\varepsilon>0$, then the closed-loop state-trajectory solutions $\left\{x_{2 n+1}\right\}$ and $\left\{x_{2 n+1}\right\}$ converge to the best proximity points $\varepsilon$ and $-\varepsilon$, respectively, if the initial condition is in $A$ and, conversely, if it is in $B$ under the sequence of stabilizing matrices $\left\{\delta_{j_{n}}^{i_{n}}\right\}$.

\subsection{Numerical example: a vector-valued discrete-time dynamic system with multiple parameterizations}

A numerical simulation of the above-presented example (3.1) is given now. Consider the discrete-time dynamic switched system described by

$$
x_{n+1}=T x_{n}=A_{\sigma(n)} x_{n}+B_{\sigma(n)} u_{n}
$$

with

$$
\begin{array}{ll}
A_{1}=\left[\begin{array}{cc}
1 & 0.1 \\
-4.019 & 0.1667
\end{array}\right], & A_{2}=\left[\begin{array}{cc}
1 & 0.1 \\
-2.4504 & 0.4369
\end{array}\right], \\
A_{3}=\left[\begin{array}{cc}
1 & 0.1 \\
-1.5256 & 0.6069
\end{array}\right], & B_{1}=10^{-3} \cdot\left[\begin{array}{ll}
0 & 0.1667
\end{array}\right]^{T}, \\
B_{2}=10^{-3} \cdot\left[\begin{array}{ll}
0 & 0.1126
\end{array}\right]^{T}, & B_{3}=10^{-4} \cdot\left[\begin{array}{ll}
0 & 0.7862
\end{array}\right]^{T}
\end{array}
$$

and $\sigma: \mathbf{N}_{0} \rightarrow\{1,2,3\}$ being the so-called switching function, which selects one of the dynamic systems subscripted by 1,2 or 3 which parameterize the time-varying system (3.12) at each discrete-time instant (or sample), $n$. This dynamic system is a simplified version of an automobile roll dynamics enhancement control system given in [15]. The switching function is assumed, for simulation purposes, to be the 1-sample periodic (cyclic) sequence $1 \rightarrow 2 \rightarrow 3 \rightarrow 1 \rightarrow 2 \rightarrow \cdots$. The following state-feedback gains are considered:

$$
\begin{aligned}
& K_{n}^{(1)}=10^{3} \cdot\left[\begin{array}{ll}
2.514 & -2.8
\end{array}\right]^{T}, \quad K_{n}^{(2)}=10^{4} \cdot\left[\begin{array}{ll}
-1 & 0.2
\end{array}\right]^{T}, \\
& K_{n}^{(3)}=10^{4} \cdot\left[\begin{array}{ll}
-1.3 & 0.8
\end{array}\right]^{T} .
\end{aligned}
$$

The control design problem can be formulated as how to select the appropriate feedback gain at each sample, $K_{n}$, from the set $\left\{K_{n}^{(1)}, K_{n}^{(2)}, K_{n}^{(3)}\right\}$ in order to guarantee the asymptotic stability of the closed-loop. For this purpose, a dynamic optimization procedure can be used. Therefore, $n$, one considers the multivalued map $x_{n+1}=T x_{n}=A_{\sigma(n)} x_{n}+B_{\sigma(n)} K_{n}^{(i)} x_{n}=$ $\left(A_{\sigma(n)}+B_{\sigma(n)} K_{n}^{(i)}\right) x_{n}$ for $i=1,2,3$ for each sample $n \in \mathbf{N}_{0}$ from $\mathbf{R}^{2}$ to $\mathbf{R}^{2}$. The Banach space $\left(\mathbf{R}^{2},\|\|\right)$ can be identified with the metric space $\left(\mathbf{R}^{2}, d\right)$ by taking the distance $d: X \times X \rightarrow$ $\mathbf{R}_{0+}$ to be the Euclidean norm. Thus, $d\left(x_{n}, 0\right)=\left\|x_{n}\right\|$ and $d\left(x_{n}, x_{n+1}\right) x=\left\|x_{n+1}-x_{n}\right\|$ so that 


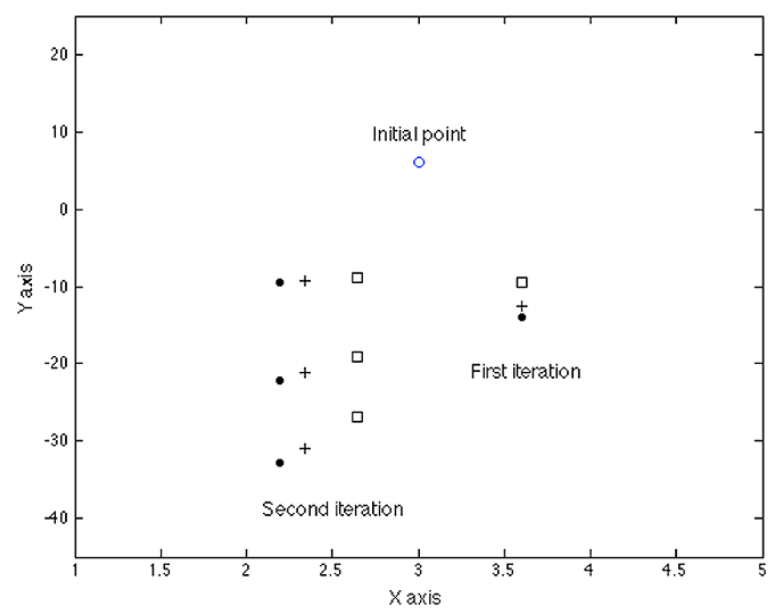

Figure 2 Graphical representation of two iterations of the multivalued map $T$.

it is direct to apply the formalism and results of Section 2. The multivalued composite map $T^{H} x_{n}=\underbrace{T \circ T \circ \cdots \circ T}_{H} x_{n}$ represents the set of reachable states starting from $x_{n}$ for all potential feedback gains $\left\{K_{n}^{(1)}, K_{n}^{(2)}, K_{n}^{(3)}\right\}$ at each sample. Figure 2 displays graphically this concept. The starting point is depicted with a circle. The application of the multivalued map $T$ to this point produces the three points (each one corresponding to one of the feedback matrices $\left.\left\{K_{n}^{(1)}, K_{n}^{(2)}, K_{n}^{(3)}\right\}\right)$, labeled as first iteration in Figure 2. A second application of $T$ generates three more points from each previous one, providing nine new points, which are depicted in Figure 2 as the second iteration. This procedure can be continued to generate the complete set of reachable states from $x_{n}$. The 'plus' symbols are used to represent the image for $K_{n}^{(1)}$, dots are used for $K_{n}^{(2)}$, while squares are used to represent the image for $K_{n}^{(3)}$.

The control algorithm generates all the images of the multivalued map $T$ and then chooses the gain $K_{n}$ in such a way that the null vector, $z=0$, is a fixed point of the multivalued map $T$. In this example, the choice $K_{n}=K_{n}^{(1)}$ for all $n \geq 0$ allows stabilizing asymptotically the system. Then, according to Proposition 3.1, all the states are bounded and the norm of the state converges to zero asymptotically as Figures 3 and 4 show.

In addition, it can be verified that the following matrices are convergent as Proposition 3.1(i) states:

$$
\begin{aligned}
& \operatorname{eig}\left(\prod_{k=1}^{2}\left[A_{\sigma(k)}+B_{\sigma(k)} K_{1}\right]\right)=\{0.3242,0.0626\} \\
& \operatorname{eig}\left(\prod_{k=1}^{3}\left[A_{\sigma(k)}+B_{\sigma(k)} K_{1}\right]\right)=\{0.0943 \pm 0.0407 j\} \\
& \operatorname{eig}\left(\prod_{k=1}^{4}\left[A_{\sigma(k)}+B_{\sigma(k)} K_{1}\right]\right)=\{0.0825,0.0077\}
\end{aligned}
$$

while the eigenvalues of the matrix product $\prod_{k=1}^{N}\left[A_{\sigma(k)}+B_{\sigma(k)} K_{1}\right]$, which describes the evolution of the discrete dynamics, converge asymptotically to zero as $N \rightarrow \infty$. 


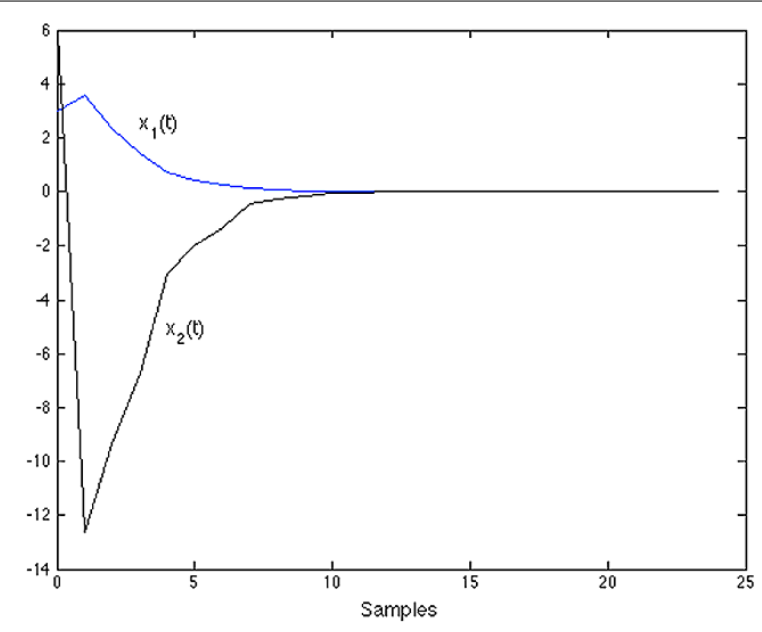

Figure 3 Evolution of the states with initial condition $x_{0}^{T}=[36]$ and $K_{n}=K_{n}^{(1)}$.

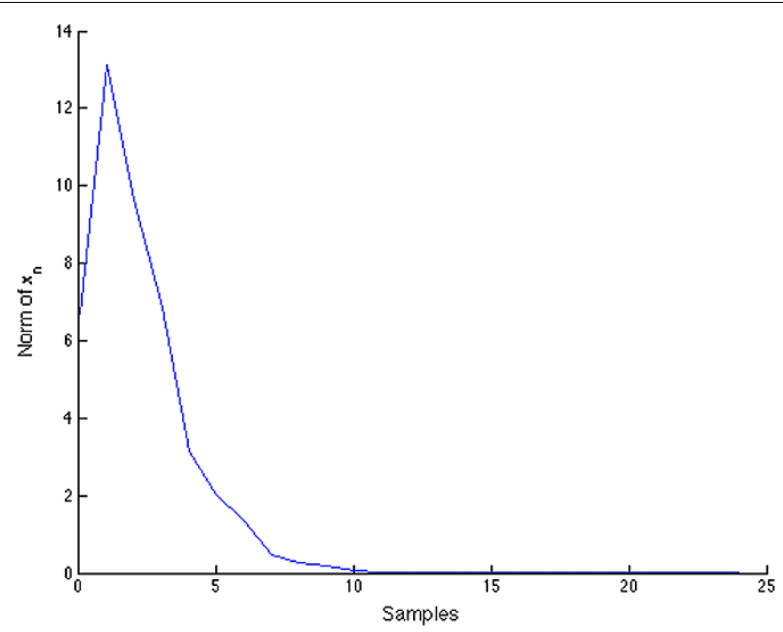

Figure 4 Evolution of the norm of the state, $\left\|x_{n}\right\|$.

\subsection{Numerical example: a scalar discrete-time dynamic system with multiple} parameterization

Now consider the controlled single-input single-output (SISO) dynamic system of the control sequence $\left\{u_{n}\right\}_{n \in \mathbf{N}_{0}}$ given by

$$
x_{n+1}=T x_{n}=x_{n}+u_{n}
$$

with the state-feedback control law $u_{n}=K_{n} x_{n}$, where $K_{n} \in\left\{K_{n}^{(1)}, K_{n}^{(2)}, K_{n}^{(3)}\right\}=\{-1.5,-1,-2\}$. This system defines the multivalued map

$$
x_{n+1}=T x_{n}=\left(1+K_{n}\right) x_{n}=\left\{\begin{array}{l}
x_{n+1}^{(1)}=\left(1+K_{n}^{(1)}\right) x_{n}, \\
x_{n+1}^{(2)}=\left(1+K_{n}^{(2)}\right) x_{n}, \\
x_{n+1}^{(3)}=\left(1+K_{n}^{(3)}\right) x_{n} .
\end{array}\right.
$$




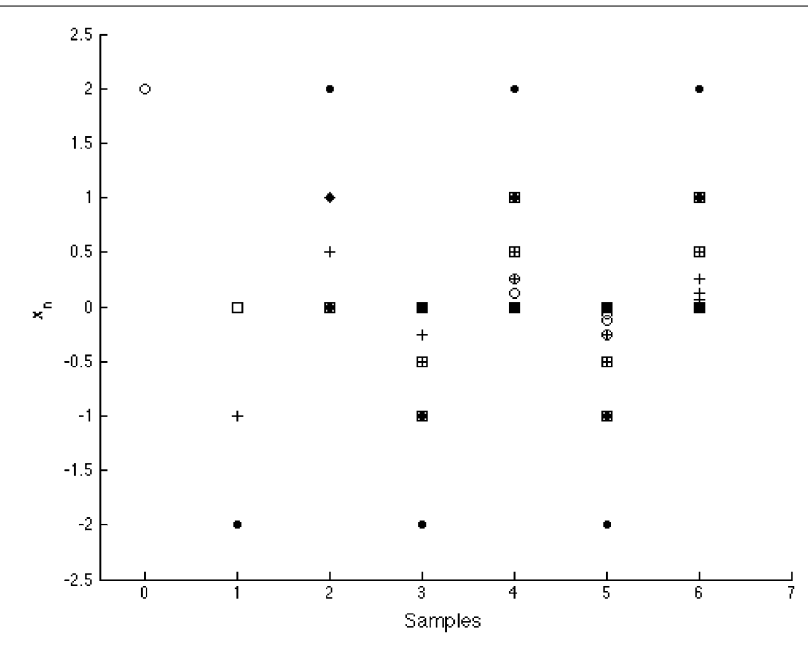

Figure 5 Several iterations of the multivalued map (3.14).

Note that (3.14) is a 2-cyclic self-mapping with non-disjoint semi-closed sets $A_{i}(i=1,2)$ of $A_{1}=-A_{2}=\mathbf{R}_{0+}=\mathbf{R}_{+} \cup\{0\}$ of $X=\mathbf{R}$ whose intersection is $\{0\}$. Consider the complete metric space $(X, d)$ which is also a Banach space $(X,\|\|)$ if the defined distance is the Euclidean norm. Thus, from each value of $x_{n}$, (3.12) generates an image set of dimension 3 , the points being labeled as $x_{n+1}^{(1)}, x_{n+1}^{(2)}$ and $x_{n+1}^{(3)}$. If the iteration process goes on, then each one of these values generates three more ones as depicted in Figure 5.

The multivalued map generates three images at $k=1$ from the starting value at $k=0$, $x_{0}=2$. Then at $k=2$ three more values are obtained from each previous one. However, note that only four different values are obtained at $k=2$ and five at $k=3$. Thus, the image of $T^{H} x_{n}$ possesses repeated values. Moreover, note that as the number of iterations increases, there are a larger number of points approaching zero since the use of the stabilizing gain $K_{n}^{(1)}$ forces some of the previously obtained points to approach zero. The particular numerical values for the first iterations showed in Figure 5 are as follows:

$$
\begin{aligned}
& x_{0}=2, \\
& x_{1}^{(1)}=-1, \quad x_{1}^{(2)}=0, \quad x_{1}^{(3)}=-2, \\
& x_{2}^{(1,1)}=0.5, \quad x_{2}^{(1,2)}=0, \quad x_{2}^{(1,3)}=1, \\
& x_{2}^{(2,1)}=0, \quad x_{2}^{(2,2)}=0, \quad x_{2}^{(2,3)}=0, \\
& x_{2}^{(3,1)}=1, \quad x_{2}^{(3,2)}=0, \quad x_{2}^{(3,3)}=2, \\
& x_{3}^{(1,1,2)}=x_{3}^{(1,3,2)}=x_{3}^{(3,1,2)}=x_{3}^{(1,2,1)}=x_{3}^{(1,2,2)}=x_{3}^{(1,2,3)}=x_{3}^{(2,1,1)}=x_{3}^{(2,1,2)}=x_{3}^{(2,1,3)}=x_{3}^{(3,3,2)} \\
& =x_{3}^{(2,2,1)}=x_{3}^{(2,2,2)}=x_{3}^{(2,2,3)}=x_{3}^{(2,3,1)}=x_{3}^{(2,3,2)}=x_{3}^{(2,3,3)}=x_{3}^{(3,2,1)}=x_{3}^{(3,2,2)}=x_{3}^{(3,2,3)}=0, \\
& x_{3}^{(1,1,1)}=-0.25, \\
& x_{3}^{(1,1,3)}=x_{3}^{(1,3,1)}=x_{3}^{(3,1,1)}=-0.5, \\
& x_{3}^{(3,1,3)}=x_{3}^{(1,3,3)}=x_{3}^{(3,3,1)}=-1, \\
& x_{3}^{(3,3,3)}=-2,
\end{aligned}
$$




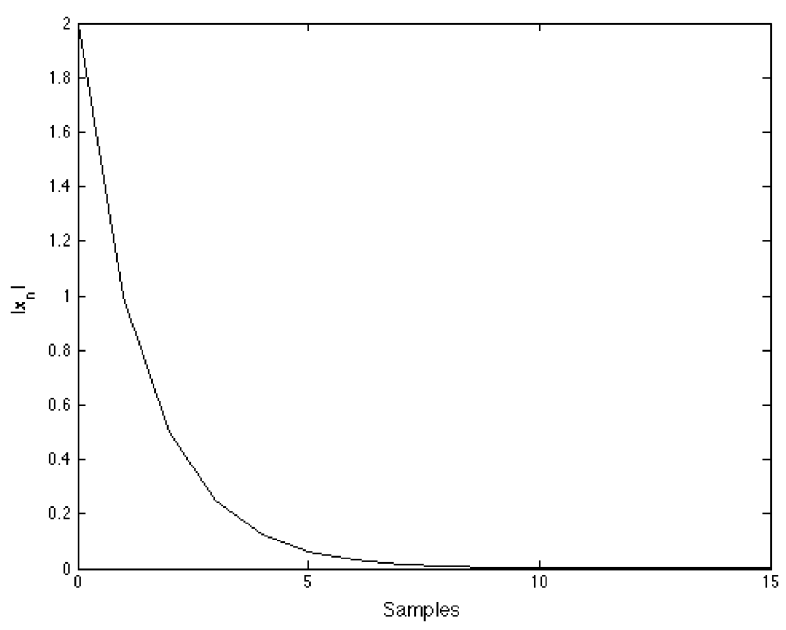

Figure 6 Evolution of the norm of $x_{n}$.

where the subscript denotes the sample while the superscript denotes the sequence of gains used to reach the point from $k=0$. System (3.11) is asymptotically stabilizable provided that at least one of the following conditions for the sequence of feedback gains $\left\{K_{n}\right\}_{n=0}^{\infty}$ is met:

(i) $\left\{K_{n}\right\}_{n=0}^{\infty}$ contains $K_{n}^{(2)}$ at least once,

(ii) $\left\{K_{n}\right\}_{n=0}^{\infty}$ contains $K_{n}^{(1)}$ an infinite (countable) number of times.

For instance, the sequence $K_{n}=K_{n}^{(1)}$ for all $n \geq 0$ satisfies the above condition (ii) and, according to Proposition 3.1, the norm of the state will converge to zero as shown in Figure 6.

\section{Competing interests}

The authors declare that they have no competing interests.

\section{Authors' contributions}

All the authors participated actively in the ellaboration of the whole paper. All authors read and approved the final manuscript.

\section{Author details}

${ }^{1}$ Institute of Research and Development of Processes, University of Basque Country, Campus of Leioa (Bizkaia) - Aptdo. 644 - Bilbao, Bilbao, 48080, Spain. ${ }^{2}$ Pt. L. M. S. Government Autonomous Postgraduate College, Rishikesh, 249201, India. ${ }^{3}$ Department of Mathematics, Semnan University, P.O. Box 35195-363, Semnan, Iran. ${ }^{4}$ Department of

Telecommunications and Systems Engineering, Universitat Autònoma de Barcelona, UAB, Barcelona, 08193, Spain. ${ }^{5}$ Department of Mathematics, Texas A\&M University-Kingsville, 700 University Blvd., Kingsville, TX 78363-8202, USA.

${ }^{6}$ Department of Mathematics, Faculty of Science, King Abdulaziz University, Jeddah, 21589, Saudi Arabia.

\section{Acknowledgements}

The first and fourth authors (M De la Sen and A Ibeas) are grateful to the Spanish Government for its support of this research through Grant DPI2012-30651, and to the Basque Government for its support of this research through Grants IT378-10 and SAIOTEK S-PE12UN015. They are also grateful to the University of Basque Country for its financial support through Grant UFI 2011/07. The second author (SL Singh) acknowledges the support by the UGC New Delhi under Emeritus Fellowship. The authors are also grateful to the referees for their valuable comments which helped to improve the manuscript.

Received: 26 July 2013 Accepted: 5 November 2013 Published: 27 Nov 2013

\section{References}

1. Đorić, D, Lazović, R: Some Suzuki-type fixed point theorems for generalized multivalued mappings and applications. Fixed Point Theory Appl. 2011, 40 (2011) doi:10.1186/1687-1812-2011-40

2. Ćirić, L: Multi-valued nonlinear contraction mappings. Nonlinear Anal. 71(7-8), 2716-2723 (2009). doi:10.1016/.na.2009.01.116

3. Ćirić, L: Fixed points for generalized multi-valued contractions. Mat. Vesn. 9(24), 265-272 (1972)

4. Gordji, ME, Cho, YJ, Ghods, S, Dehkordi, MH: Coupled fixed-point theorems for contractions in partially ordered metric spaces and applications. Math. Probl. Eng. 2012, Article ID 150363 (2012). doi:10.1155/2012/150363 
5. Singh, SL, Mishra, SN, Jain, S: Round-off stability of multi-valued maps. Fixed Point Theory Appl. 2012, 12 (2012). doi:10.1186/1687-1812-2012-12

6. Singh, SL, Mishra, SN, Chugh, R, Kamal, R: General common fixed point theorems and applications. J. Appl. Math. 2012, Article ID 902312 (2012). doi:10.1155/2012/902312

7. Laowang, W, Panyanak, B: Common fixed points for some generalized multivalued nonexpansive mappings in uniformly convex metric spaces. Fixed Point Theory Appl. 2011, 20 (2011). doi:10.1186/1687-1812-2011-20

8. Yao, Y, Noor, MA, Liou, YC, Kang, SM: Iterative algorithms for general multivalued variational inequalities. Abstr. Appl. Anal. 2012, Article ID 768272 (2012). doi:10.1155/2012/768272

9. Khandani, H, Vaezpour, SM, Sims, B: Common fixed points of generalized multivalued contraction in complete metric spaces. J. Comput. Anal. Appl. 13(6), 1025-1038 (2011)

10. Abbas, M: Coincidence points of multivalued $f$-almost nonexpansive mappings. Fixed Point Theory 13(1), 3-10 (2012)

11. Rezapour, SH, Amiri, P: Fixed point of multivalued operators on ordered generalized metric spaces. Fixed Point Theory 13(1), 173-178 (2012)

12. Petrusel, A, Petrusel, G: Multivalued Picard operators. J. Nonlinear Convex Anal. 13(1), 157-171 (2012)

13. Petru, TP, Petrusel, A, Yao, JC: Ulam-Hyers stability for operational equations and inclusions via nonself operators. Taiwan. J. Math. 15(5), 2195-2212 (2011)

14. Gordji, ME, Baghani, H, Cho, YJ: Coupled fixed point theorems for contractions in intuitionistic fuzzy normed spaces. Math. Comput. Model. 54(9-10), 1897-1906 (2011)

15. Nashine, HK, Shatanawi, W: Coupled common fixed point theorems for a pair of commuting mappings in partially ordered complete metric spaces. Comput. Math. Appl. 62, 1984-1993 (2011)

16. Solmaz, S, Shorten, R, Wulff, K, Cairbre, FO: A design methodology for switched discrete time linear systems with applications to automotive roll dynamics control. Automatica 44(9), 2358-2363 (2008)

17. Sahu, DR, Liu, ZQ, Kang, SM: Existence and approximation of fixed points of nonlinear mappings in spaces with weak uniform normal structure. Comput. Math. Appl. 64(4), 672-685 (2012)

18. Shatanawi, W, Postolache, M: Common fixed point results of mappings for nonlinear contraction of cyclic form in ordered metric spaces. Fixed Point Theory Appl. 2013, 60 (2013). doi:10.1186/1687-1812-2013-60

19. Inchan, I: Viscosity iteration method for generalized equilibrium points and fixed point problems of finite family of nonexpansive mappings. Appl. Math. Comput. 219(6), 2949-2959 (2012)

20. Hussain, N, Pathak, HK: Common fixed point and approximation for $\mathrm{H}$-operator pair with applications. Appl. Math. Comput. 218(2), 11217-11225 (2012)

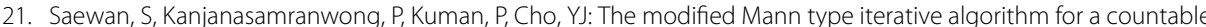
family of totally quasi- $\varphi$-asymptotically nonexpansive mappings by the hybrid generalized $f$-projection method. Fixed Point Theory Appl. 2013, 63 (2013). doi:10.1186/1687-1812-2013-63

22. Nashine, HK, Khan, MS: An application of fixed point theorem to best approximation in locally convex space. Appl. Math. Lett. 23(2), 121-127 (2010)

23. Shen, T, Yuan, Z: Stability criterion for a class of fixed-point digital filters using two's complement arithmetic. Appl. Math. Comput. 219(9), 4880-4883 (2013)

24. Reich, S: Some remarks concerning contraction mappings. Can. Math. Bull. 14, 121-124 (1971)

25. De la Sen, M: Stable iteration procedures in metric spaces which generalize a Picard-type iteration. Fixed Point Theory Appl. 2010, Article ID 953091 (2010). doi:10.1155/2010/953091

26. Zabczyk, J: Mathematical Control Theory. An Introduction. Modern Birkhäuser Classics. Birkhäuser, Boston (1992)

27. Imdad, M, Erduran, A: Suzuki-type generalization of Chatterjea contraction mappings on complete partial metric spaces. J. Oper. 2013, Article ID 923843 (2013)

28. Kadelburg, Z, Nashine, HK, Radenović, S: Coupled fixed points in partial metric spaces. J. Adv. Math. Stud. 6(1), 159-172 (2013)

29. Nashine, HK, Kadelburg, Z, Radenović, S: Fixed point theorems via various cyclic contractive conditions in partial metric spaces. Publ. Inst. Math. 107, 69-93 (2013)

30. Nashine, HK, Kadelburg, Z: Cyclic contractions and fixed point results via control functions on partial metric spaces. Int. J. Anal. 2013, Article ID 726387 (2013)

31. Kadelburg, Z, Nashine, HK, Radenović, S: Common coupled fixed point results in partially ordered G-metric spaces. Bull. Math. Anal. Appl. 4(2), 51-63 (2012)

32. De la Sen, M: Some results on fixed and best proximity points of multivalued cyclic self-mappings with a partial order Abstr. Appl. Anal. 2013, Article ID 968492 (2013). doi:10.1155/2013/968492

33. Kikkawa, M, Suzuki, T: Three fixed point theorems for generalized contractions with constants in complete metric spaces. Nonlinear Anal., Theory Methods Appl. 69(9), 2942-2949 (2008)

34. Enjouji, Y, Nakanishi, M, Suzuki, T: A generalization of Kannan's fixed point theorem. Fixed Point Theory Appl. 2009, Article ID 192872 (2009). doi:10.1155/2009/192872

35. Eldred, AA, Veeramani, P: Existence and convergence of best proximity points. J. Math. Anal. Appl. 323(2), 1001-1106 (2006)

36. De la Sen, M: Linking contractive self-mappings and cyclic Meir-Keeler contractions with Kannan self-mappings. Fixed Point Theory Appl. 2010, Article ID 572057 (2010). doi:10.1155/2010/572057

37. Karpagam, S, Agrawal, S: Best proximity point theorems for $p$-cyclic Meir-Keller contractions. Fixed Point Theory Appl. 2009, Article ID 197308 (2009). doi:10.1155/2009/197308

38. Subrahmanyam, PV: Completeness and fixed points. Monatshefte Math. 80(4), 325-330 (1975)

39. Suzuki, T: A generalized Banach contraction principle that characterizes metric completeness. Proc. Am. Math. Soc. 136(5), 1861-1869 (2008)

40. Sintunavarat, $W$, Kuman, $\mathrm{P}$ : The existence theorems of an optimal approximate solution for generalized proximal contraction mappings. Abstr. Appl. Anal. 2013, Article ID 375604 (2013). doi:10.1155/2013/375604

41. De la Sen, M, Ibeas, A: Stability results for switched linear systems with constant discrete delays. Math. Probl. Eng. 2008, Article ID 543145 (2008). doi:10.1155/2008/543145

42. De la Sen, M, Ibeas, A: On the stability properties of linear time-varying unforced systems involving switches between parameterizations from topologic considerations via graph theory. Discrete Appl. Math. 155(1), 7-25 (2007) 
43. De la Sen, M: Robust stable pole-placement based adaptive control of continuous linear systems with two parametrical estimation schemes. J. Franklin Inst. Eng. Appl. Math. 341(3), 251-254 (2004)

44. De la Sen, M, Karapinar, E: On best proximity points of generalized semi-cyclic impulsive self-mappings. Applications to impulsive differential and difference equations. Abstr. Appl. Anal. 2013, Article ID 505487 (2013). doi:10.1155/2013/505487

45. Larson, RE: State Increment Dynamic Programming. Modern Analytic and Computational Methods in Science and Mathematics. Elsevier, Amsterdam (1969)

46. Ratchagit, M, Ratchagit, K: Asymptotic stability and stabilization of fixed points for iterative sequence. Int. J. Res. Rev. Comput. Sci. 2(4), 987-989 (2011)

10.1186/1687-1812-2013-324

Cite this article as: De la Sen et al.: Best proximity and fixed point results for cyclic multivalued mappings under a generalized contractive condition. Fixed Point Theory and Applications 2013, 2013:324

\section{Submit your manuscript to a SpringerOpen ${ }^{\circ}$ journal and benefit from:}

- Convenient online submission

- Rigorous peer review

- Immediate publication on acceptance

- Open access: articles freely available online

- High visibility within the field

- Retaining the copyright to your article 